\title{
PBX3/MEK/ERK1/2/LIN28/let-7b positive feedback loop enhances mesenchymal phenotype to promote glioblastoma migration and invasion
}

Xiupeng $\mathrm{Xu}^{1+}$, Zhongyuan Bao ${ }^{1+}$, Yinlong Liu ${ }^{2+}$, Kuan Jiang ${ }^{3+}$, Tongle Zhi $^{1}$, Dong Wang ${ }^{1}$, Liang Fan ${ }^{1}$, Ning Liu ${ }^{1}$ and Jing $\mathrm{Ji}^{\mathrm{i}^{*}}$

\begin{abstract}
Background: Brain invasion by glioblastoma (GBM) determines recurrence and prognosis in patients, which is, in part, attributed to increased mesenchymal transition. Here, we report evidence favoring such a role for the Pre-Bcell leukemia homebox (PBX) family member PBX3.

Methods: Western blot, immunohistochemistry, qRT-PCR and datasets mining were used to determined proteins or genes expression levels. Wound-healing and transwell assays were used to examine the invasive abilities of GBM cells. Dual-luciferase reporter assays were used to determine how let-7b regulates PBX3. Chromatinimmunoprecipitation (ChIP) and rescue experiments were performed to investigate the involved molecular mechanisms. Orthotopic mouse models were used to assess the role of PBX3 in vivo.

Results: We found that PBX3 expression levels positively correlated with glioma mesenchymal markers. Ectopic expression of PBX3 promoted invasive phenotypes and triggered the expression of mesenchymal markers, whereas depletion of PBX3 reduced GBM cell invasive abilities and decreased the expression of mesenchymal markers. In addition, inhibition of PBX3 attenuated transforming growth factor- $\beta$ (TGF $\beta$ )-induced GBM mesenchymal transition. Mechanistic studies revealed that PBX3 mediated GBM mesenchymal transition through activation of MEK/ERK1/2, leading to increased expression of LIN28 by c-myc. Increased LIN28 inhibited let-7b biogenesis, which then promoted the pro-invasive genes, such as HMGA2 and IL-6. Furthermore, let-7b suppressed PBX3 by directly targeting 3'-UTR of PBX3. Thus, repressed let-7b by PBX3 amplifies PBX3 signaling and forms a positive feedback loop to promote GBM mesenchymal transition.
\end{abstract}

Conclusions: These data highlight the importance of PBX3 as a key driver of mesenchymal transition and potential therapeutic target.

Keywords: PBX3, Mesenchymal transition, ERK1/2, LIN28/let-7b, C-myc, GBM

\footnotetext{
*Correspondence: jijing@njmu.edu.cn

${ }^{+}$Xiupeng Xu, Zhongyuan Bao, Yinlong Liu and Kuan Jiang contributed equally to this work.

'Department of Neurosurgery, the First Affiliated Hospital of Nanjing Medical

University, Guangzhou Road 300, Nanjing, Jiangsu, China

Full list of author information is available at the end of the article
}

C The Author(s). 2018 Open Access This article is distributed under the terms of the Creative Commons Attribution 4.0 International License (http://creativecommons.org/licenses/by/4.0/), which permits unrestricted use, distribution, and reproduction in any medium, provided you give appropriate credit to the original author(s) and the source, provide a link to the Creative Commons license, and indicate if changes were made. The Creative Commons Public Domain Dedication waiver (http://creativecommons.org/publicdomain/zero/1.0/) applies to the data made available in this article, unless otherwise stated. 


\section{Background}

Glioblastoma (GBM) is the most common and lethal malignant brain tumor in adults [1]. Despite recent advances in therapeutic strategies, including surgical resection followed by radiotherapy and temozolomide chemotherapy, the average survival of patients with GBM is approximately 14 months after the initial diagnosis $[2,3]$. This dismal prognosis may be partly attributed to the highly invasive nature of GBM, which favors its infiltration into the surrounding normal brain parenchymal, making total surgical resection impossible $[4,5]$. Thus, there is an urgent need to identify therapies that decrease GBM invasion and increase patient survival.

The inability to define different patient outcomes based on the traditional histopathological classification suggests a large problem in our understanding of the classification of GBM [6]. To better understand determinants of GBM malignant progression, genomic- and genetic-based molecular stratification of GBM were performed [7]. Although inconsistence were observed among different classification studies, the proneural and mesenchymal subtypes were highly consistent in literatures [8-11]. The proneural subtype is characterized by the high expression of genes involved in neurogenesis, and is associated with a favorable outcome [10]. By contrast, the mesenchymal subtype shows high expression of genes correlated with invasion, motility and stemness, and is associated with poor clinical outcomes [12, 13]. Importantly, proneural-to-mesenchymal transition (PMT) was frequently observed upon GBM relapse [14, 15]. Subsequent studies demonstrated that this process is mediated by several key transcriptional factors, such as nuclear factor $\kappa B$ (NF- $\mathrm{kB})$ [16], Snail Family Transcriptional Repressor 1 (SNAI1) [17] and Signal Transducer and Activator of Transcription 3 (STAT3) [15]. However, detailed molecular mechanisms that promote PMT remain ambiguous. Given the malignant behaviors of mesenchymal GBM, uncovering the underlying molecular mechanisms that responsible for maintaining mesenchymal phenotype is urgently needed for targeted therapies.

Pre-B-cell leukemia homebox 3 (PBX3) was first identified in 1991 by Monic et al. [18], which belongs to the three-amino acid-loop-extension family of homeodomain transcription factors. Subsequent studies showed that PBX3 is overexpressed in various human cancers, including prostate cancer [19], colorectal cancer [20], gastric cancer [21], hepatocellular carcinoma [22], and multiple myeloma [23]. Functional studies revealed that upregulated PBX3 promotes cancer cell malignant behaviors, such as proliferation, migration, invasion, cell cycle progression, drug resistance and cancer stemness [21-24]. Moreover, survival analysis demonstrated that PBX3 expression is predictive of poor prognosis in some malignancies [20, 22, 25]. Our group recently reported that PBX3 is overexpressed in GBM and promotes GBM migration, invasion, proliferation and cell cycle progression $[26,27]$. As PMT is associated with malignant phenotypes of GBM, we hypothesized that upregulated PBX3 might be involved in promoting PMT in GBM.

Aberrant expression of LIN28/let-7 axis has been well documented in various malignancies, including GBM [28]. Experimentally, LIN28/let-7 axis has been shown to induce cancer cell invasive phenotypes and mensenchymal transition by regulating various let-7 targets, such as high mobility group A2 (HMGA2) [29, 30]. Importantly, LIN28/let-7 axis represents a critical downstream target of MEK/ERK1/2 pathway and mediates ERK1/2-driven mesenchymal transition [30]. As PBX3 activates MEK/ ERK1/2 pathway to promote malignant phenotypes in some cancers [25], we hypothesized that PBX3 may promote GBM mesenchymal phenotype through MEK/ ERK1/2 pathway mediated LIN28/let-7 axis activation.

Here, we characterized the role of PBX3 in regulating PMT process. Our results demonstrated that upregulation/downregulation of PBX3 increases/decreases mesenchymal phenotype of GBM. Mechanically, we showed that PBX3 enhances mesenchymal phenotype of GBM through a positive feedback loop involving activation of MEK, ERK1/2, c-myc, and LIN28, leading to inhibition of the let-7b expression and upregulation of its targets. Finally, we found that PBX3 is required for TGF- $\beta$-induced GBM mesenchymal transition.

\section{Methods}

\section{Database mining and human tissue samples}

Glioma gene expression data were downloaded from three datasets: Repository for Molecular Brain Neoplasia Data (Rembrandt; http://caintegratorinfo.nci.nih.gov/re mbrandt); and the National Center for Biotechnology Information Gene Expression Omnibus (NCBI-GEO) datasets GSE4290 and GSE59612 (http://www.ncbi.nlm.g ov.geo/; accession nos. GSE4290 and GSE59612). Forty-five GBM samples were collected from the Department of Neurosurgery, The First Affiliated Hospital of Nanjing Medical University between 2011 and 2014. Informed consent for the use of samples was obtained from all patients. Our study was approved by the institutional review board and ethics committee of Nanjing Medical University.

\section{Gene set enrichment analysis (GSEA)}

The GSEA was performed using software downloaded from the Broad Institute (http://www.broadinstitute.org/ gsea/index.jsp) with $\mathrm{H}$ (hallmark gene sets) collection. We divided the GBM cohort in Rembrandt into two groups with high PBX3 expression or low PBX3 expression. The Gene Cluster Text file (.gct) was generated from the Rembrandt dataset. The Categorical Class file 
(.cls) was prepared based on the PBX3 mRNA levels of GBM patients in the Rembrandt dataset. Using a permutation test at 1000 times, we finally identified gene signatures that were enriched in the PBX3 high expression group.

\section{Cell culture, transfection and drug treatment}

U87 and U251 cells were purchased from the American Type Culture Collection (ATCC, Manassas, VA). Cells were cultured in Dulbecco's modified Eagle's medium (DMEM; Hyclone, USA) supplemented with $10 \%$ fetal bovine serum (Gibco, Invitrogen, Carlsbad, CA, USA) at $37{ }^{\circ} \mathrm{C}$ in a humidified atmosphere with $5 \% \mathrm{CO}_{2}$.

Lentiviruses carrying PBX3 or vectors or siRNA-PBX3 or siRNA-NC were purchased from Genepharma (Shanghai, China). Stable U87 and U251 cells were established by lentiviral infection and puromycin selection as manufacturer's protocol. Although, our previous study showed that PBX3 protein levels in $\mathrm{H} 4$ and U118 cell lines were significantly lower than that in U87 and U251 cells [27], we did not select H4 and U118 cells for overexpression studies. The main reason is the low ovexpression efficiency of PBX3 in both $\mathrm{H} 4$ and $\mathrm{U} 118$ cells. Let-7b mimic and inhibitor were purchased from RiboBio (Guangzhou, China) and transfected into cells using Lipofectamine RNAiMAX Reagent (Life Technologies, Grand Island, NY) according to manufacturer's protocol. siRNAs or plasmids were synthesized by Genepharma and transfected into cells using Lipofectamine 2000 Transfection Reagent (Invitrogen).

Recombinant human transforming growth factor- $\beta$ (TGF $\beta$ ), U0126 and phorbol 12-myristate 13-acetate (PMA) were purchased from Sigma, and diluted according to each manufacturer's protocol. The dose of each drug was used as previously described [17, 31].

\section{RNA extraction and quantitative real-time polymerase chain reaction (qRT-PCR)}

Total RNA extraction and qRT-PCR for mRNA were performed as previously described [26]. Primers used in this study were listed as follows: PBX3 forward $5^{\prime}$-CA AGTCGGAGCCAATGTG-3' and reverse 5' -ATGTAG CTCAGGGAAAAGTG-3'; N-cadherin forward 5'-GAA GGAGGTGGGGAGGAAGATA-3' and reverse $5^{\prime}$-GGT GGTCTCTGACGAGGTAAACA-3'; ZEB1 forward 5' AGTTTACCTTCCAGCAGCCCTAC-3'; and reverse 5'-AGCTCTTCTGCACTTGGTTGTG-3'; Slug forward 5'-AGACCCCCATGCCATTGAAG-3' and reverse $5^{\prime}$-G GCCAGCCCAGAAAAAGTTG-3'; CD44 forward 5'-C ACAACAACACAAATGGCTG-3' and reverse $5^{\prime}$-CA ATGCCTGATCCAGAAAAA-3'; IL-6 forward 5'-TCCA GAACAGATTTGAGAGTAGTG-3' and reverse $5^{\prime}$-GC ATTTGTGGTTGGGTCAGG-3'; HMGA2 forward 5' CACTTCAGCCCAGGGACAAC-3' and reverse 5'-G CCTCTTGGGCGTTTTTCTC-3'; $\beta$-actin forward 5'-G
TGATCTCCTTCTGCATCCTGT-3' and reverse 5' -CC ACGAAACTACCTTCAACTCC-3'; qRT-PCR for let-7b was performed using commercially available TaqMan ${ }^{\circ}$ MicroRNA Assays (\#4373168, Applied Biosystems, Darmstadt, Germany) according to manufacturer's protocol. The specificity of PCR was confirmed by melting curves and PCR product sequencing.

\section{Western blot analysis}

Protein extraction, quantification and immunoblotting were performed as our previously described [27]. The antibodies used in this study were: PBX3 (1:500, ab109173), N-cadherin (1:750, ab18203), ZEB1 (1:500, ab180905), Slug (1:750, ab180714), CD44 (1:750, ab51037), c-myc (1:500, ab32072), LIN28 (1:500, ab46020) and $\beta$-actin (1:2000, ab8226) all from Abcam (Cambridge, UK). HMGA2 (1:1000, no. 8179), STAT3 (1:1000, no. 9139), phospho-STAT3 (1:500, no. 9145), MEK1/2 (1:1500, no. 4694), phospho-MEK1/2 (1:1000, no. 4370), ERK1/2 (1:2000, no. 9194,) and phospho-ERK1/2 (1:1000, no. 4370) all from Cell Signaling Technologies (Danvers, MA, USA).

\section{In vitro cell migration and invasion assays}

The invasive capabilities of glioma cells were determined as our previously reported [26].

\section{Immunohistochemsitry}

Tissues were fixed in $4 \%$ paraformaldehyde for two $48 \mathrm{~h}$ and paraffin embedded in a regular way. Tissue sections $(4 \mu \mathrm{m})$ were treated and stained with the following antibodies: PBX3 (1:75, ab109173), N-cadherin (1:100, ab18203), ZEB1 (1:150, ab180905), Slug (1:150, ab180714), CD44 (1:100, ab51037).

\section{Quantitative chromatin immunoprecipitation analysis}

Chromatin-immunoprecipitation (ChIP) assays were carried out using a ChIP Assay Kit (\#17408, Millipore) according to the manufacturer's protocol. The chromatin fragments were immunoprecipitated with $2 \mu \mathrm{g}$ of antibodies against either c-myc (ab32) or Jun (ab31419). The primer sequences used in this study were as follows: LIN28 (c-myc) forward 5'-GGGAGGGCCCATTCAT TTC-3' and reverse 5'-GGGTCCCCAAAGCAGATAC A-3'; Wnt5a (c-myc) forward 5'-GTCGGGAAGTGGTC AAGGTT-3' and reverse 5'-AAGTGCCAGAGACAG ATGCT-3'; CyclinD1 (Jun) forward 5'-GTCCCAGGC AGAGGGGAC-3' and reverse 5'-CGGCAATTTAAC CGGGAGA-3'; $\beta$-globin (negative control) forward 5 '-A GTGCCAGAGCCAAGGA-3' and reverse 5'-CAGGGT GAGGTCTAAGTGATGACA-3'; and rRNA (internal control) forward 5'-ATTAGTCAGCGGAGGAAAAGAA AC-3' and reverse 5' -TCGCCGTTACTGAGGGAATC-3' . 


\section{Luciferase reporter assay}

To determine whether let-7b directly binds to the PBX3 3 '-UTR, dual luciferase reporter assays were conducted. Wild-type (WT) and mutated putative let-7b-binding sites were amplified and cloned into the $\mathrm{XbaI}$ site of a pGL3 control vector (Invitrogen). The following reporter assays were performed in a regular way as our previously described [26].

\section{Determination of interleukin- 6 concentrations in supernatant of cultured cells}

The collected supernatant was serially diluted, and levels of IL- 6 of different groups were measured by enzyme-linked immunosorbent assay (ELISA; R\&D Systems Inc., Minneapolis, MN).

\section{Orthotopic GBM model}

Female Bagg albino (BALB)/c nude mice at 5 weeks were purchased from the Shanghai Experimental Animal Center of the Chinese Academy of Sciences and maintained in specific pathogen-free conditions for 1 week. To established intracranial GBMs, LV-siRNA-NC- or LV-siRNA-PBX3-transfected U87 cells $\left(2.5 \times 10^{5}\right)$ were stereotactically injected (12 mice for each group). Three weeks later, all mice were killed by rapid decapitation and brains were extracted and stored in liquid nitrogen. All experimental procedures were in accordance with the Guide for the Care and Use of Laboratory Animals of the National Institutes of Health and approved by the Nanjing Medical University Animal Experimental Ethics Committee.

\section{Statistical analysis}

Statistical analyses were performed using GraphPad Prism 5.0 (GraphPad Software, Inc., LaJolla, CA, USA). Data were presented as mean \pm SD. The significance of differences between groups was tested by two-tailed Student's $t$-test. Correlations between PBX3 and N-cadherin, ZEB1, Slug, CD44, let-7b, HMGA2, and IL-6 expressions were analyzed with Pearson's correlation method. Values of $p<0.05$ were considered statistically significant.

\section{Results}

PBX3 is upregulated in mesenchymal gliomas and promotes glioma migration and invasion via enhancing mesenchymal transition

To evaluate the expression profiles of PBX3 between mesenchymal and proneural gliomas, mRNA data from Rembrandt, GSE4290 and GSE59612 were analyzed. Results showed that PBX3 expression was increased in mesenchymal gliomas compared with proneural gliomas (Fig. 1a-c). Using the expression data from the Rembrandt dataset, we performed GSEA to verify whether we could detect some hallmarks of cancer, including mesenchymal transition,in the patients with high-level of PBX3. Our results showed that hallmarks, including "Epithelial_Mesenchymal_Transition", were significantly enriched in the patients with high-level of PBX3 (Fig. 1d). We chose four mesenchymal transition markers (N-cadherin, ZEB1, Slug, and CD44) from the gene list identified by GSEA, which are reported to be involved in glioma mesenchymal transition [6, 32-35], and analyzed the relationship between these markers and PBX3 expression. Pearson correlation analyses using mRNA data from Rembrandt dataset were performed. Results showed significant and positive correlations between PBX3 and N-cadherin, ZEB1, Slug and CD44 (Additional file 1: Figure S1A-D). To further confirm our observations, we measured PBX3 and mesenchymal markers mRNA levels in 45 GBM samples by qRT-PCR and observed high expression levels of N-cadherin, ZEB1, Slug and CD44 in GBMs with high PBX3 expression $(n=23)$ than those with low PBX3 expression (Additional file 1: Figure S1E-H). Having demonstrated that PBX3 is upregulated in mesenchymal gliomas and correlated with mesenchymal markers, we wondered whether PBX3 is required for maintenance of mesenchymal phenotype of GBM cells. To achieve this goal, we ectopically expressed PBX3 using lentiviral vectors and knocked down PBX3 expression using lentiviral siRNAs in U87 and U251 cells (Fig. 1e and h). Results showed that overexpression of PBX3 significantly promoted a fibroblast-like morphology and enhanced the expression of mesenchymal markers (Fig. $1 \mathrm{f}$ and g). By contrast, knockdown of PBX3 triggered a cobblestone-like morphology and decreased the expression of mesenchymal markers (Fig. $1 \mathrm{i}$ and j). On the basis of the mesenchymal promoting role of PBX3 in glioma, we focused on migration and invasion assays. As expected, PBX3 overexpression promoted glioma migration and invasion, while PBX3 downregulation inhibited glioma migration and invasion (Additional file 2: Figure S2). Collectively, these results indicated that PBX3 is associated with mesenchymal transition in gliomas.

\section{PBX3 is required for the TGF- $\beta$-induced mesenchymal transition}

TGF- $\beta$ is a master regulator of mesenchymal transition in GBM and induces the expression of mesenchymal genes, such as N-cadherin, ZEB1, Slug and CD44 [17, 36, 37]. Moreover, GSEA results showed that GBMs with high-level of PBX3 were enriched for the TGF- $\beta$ signaling (Additional file 3: Figure S3). Therefore, we decided to explore the role of PBX3 in response to TGF- $\beta$. First, we examined the expression of PBX3 mRNA and protein upon exposure to TGF- $\beta$. The levels of PBX3 mRNA and protein in U87 and U251 cells were significantly 


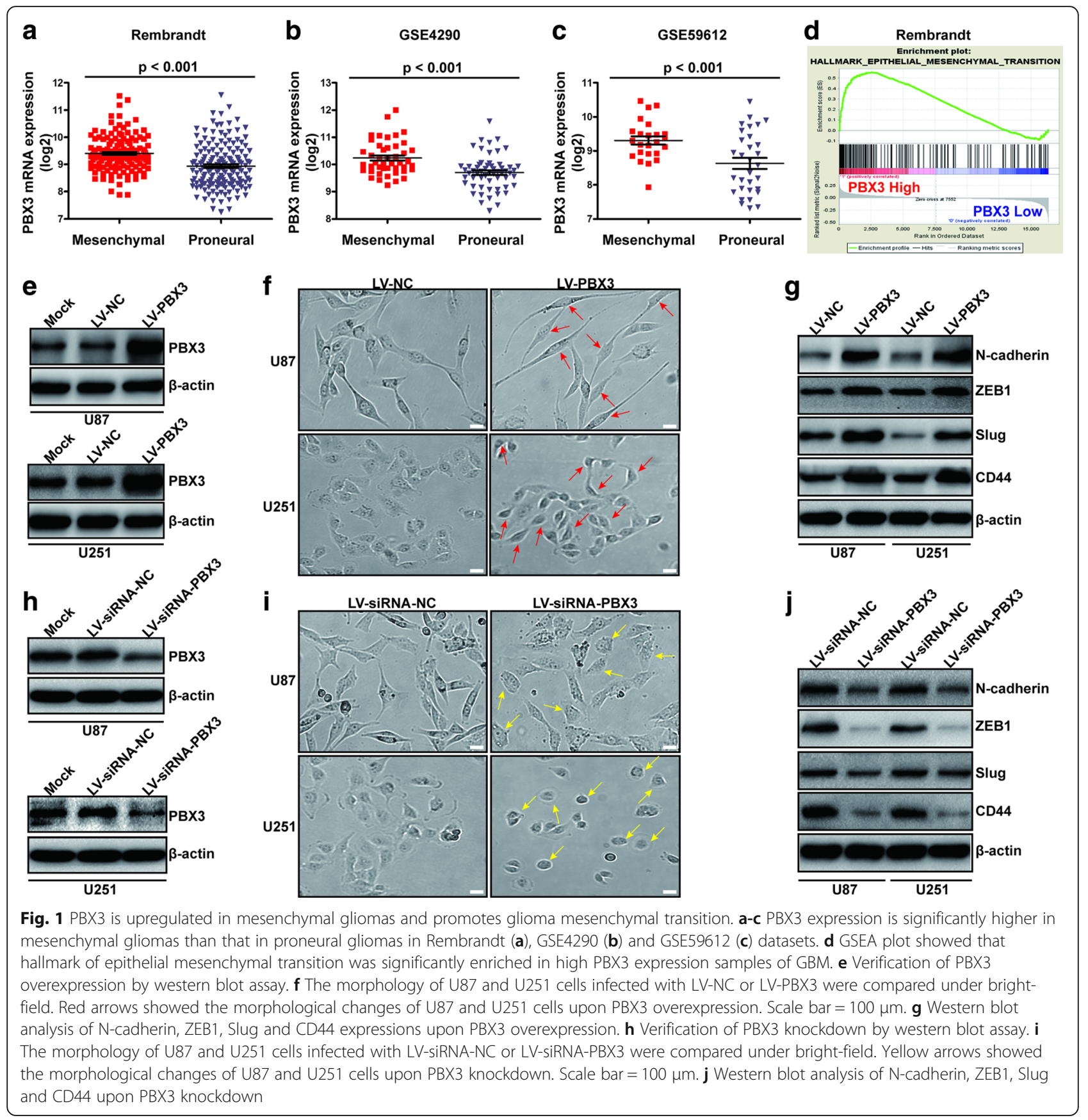

upregulated during the 48-h post-incubation period (Fig. 2a and b). Next, we tested the effect of PBX3 downregulation on the TGF- $\beta$ induced upregulation of $N$-cadherin, ZEB1, Slug and CD44. TGF- $\beta$ induced upregulation of N-cadherin, ZEB1and CD44 were completely antagonized, whereas the accumulation of Slug was partially reversed by the knockdown of PBX3 (Fig. 2c). Finally, would-healing and transwell assays demonstrated that TGF- $\beta$-induced GBM cells migration and invasion were significantly decreased by PBX3 knockdown (Fig. $2 \mathrm{~d}$ and e). In summary, these results demonstrated that PBX3 is required for the TGF- $\beta$-induced mesenchymal transition in GBM cells.

\section{LIN28/let-7b axis is required for PBX3-induced} mesenchymal transition and for increased migration and invasion

To confirm our hypothesis that PBX3 promotes glioma mesenchymal transition and then triggers migration and invasion via activating LIN28/let-7 axis, we first measured the expression of LIN28 and let-7 upon PBX3 overexpression. Results showed that overexpression of 


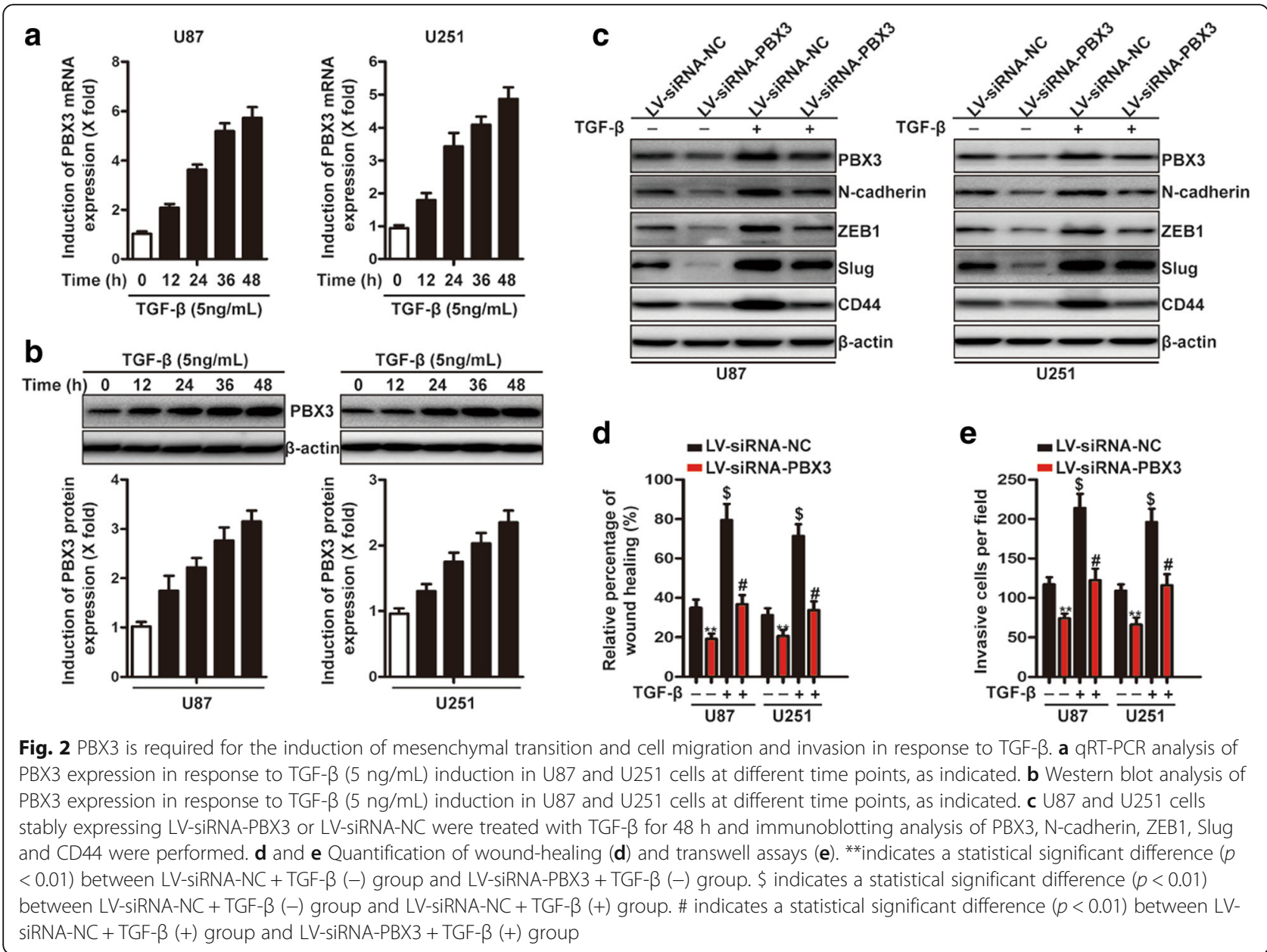

PBX3 increased LIN28 protein levels while decreased let-7 levels, with let-7b being the most downregulated one (Fig. 3a, Additional file 4: Figure S4 and Additional file 5: Figure S5A), suggesting that $\mathrm{PBX} 3$ positively regulates LIN28/let-7 axis. As let-7b represents the most significantly downregulated let-7 family microRNAs upon PBX3 overexpression, we selected let-7b for the following experiments. Next, we tested whether PBX3-driven GBM mesenchymal phenotype was dependent on LIN28/ let-7b axis. LIN28 knockdown prevented PBX3-mediated increases in cell migration and invasion and reserved PBX3-induced mesenchymal phenotype (Fig. $3 \mathrm{~b}$ and $\mathrm{c}$ and Additional file 5: Figure S5B and C). Consistently, overexpression of let-7b reversed PBX3-driven GBM cell migration, invasion and mesenchymal transition (Fig. 3d-f and Additional file 5: Figure S5D-F). To further confirm our hypothesis, we also measured LIN28 and let-7b expression under PBX3 knockdown conditions. Results showed that downregulation of PBX3 decreased LIN28 protein levels and increased let-7b expressions(Fig. 3g and Additional file 5: Figure S5G). As expected, overexpression of LIN28 or inhibition of let-7b expression reversed PBX3 downregulation-induced decreased cell migration, invasion and mesenchymal phenotype (Fig. 3h-l and Additional file 5: Figure S5H-L). Collectively, these results demonstrated that LIN28/let-7b axis is required for PBX3-driven mesenchymal transition and for increased migration and invasion.

\section{PBX3 activates LIN28/let-7b axis via ERK1/2 and c-myc- dependent mechanisms}

Having demonstrated that PBX3 activates LIN28/let-7b pathway in GBM, we next investigated whether PBX3 regulates LIN28/let-7b axis via ERK1/2-dependent mechanism as our previously expected. To achieve this goal, we need to investigate whether MEK/ERK1/2 pathway mediated PBX3-driven GBM mesenchymal transition. First, we determined the changes in expression of phospho-MEK, MEK, phospho-ERK1/2 and ERK1/2 in GBM cells with altered expression of PBX3. As expected, phospho-MEK and phospho-ERK1/2 expression were increased after overexpression of PBX3 and decreased upon PBX3 downregulation (Fig. 4a and d). These data clearly demonstrated that PBX3 could activate MEK/ 
a

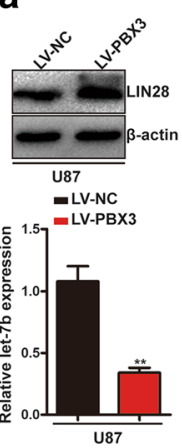

C

- LV-NC + SiRNA-NC

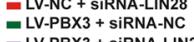

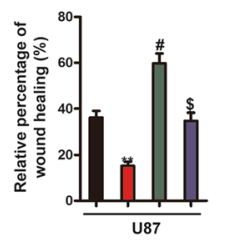

d

- LV-NC + pre-miR-NC $=$ LV-PBX3 + pre-miR-NC - LV-PBX3 + pre-let-7b

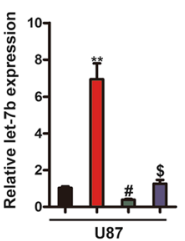

b
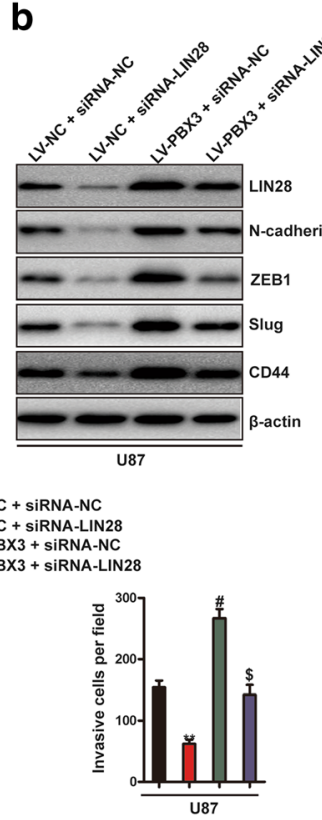

e

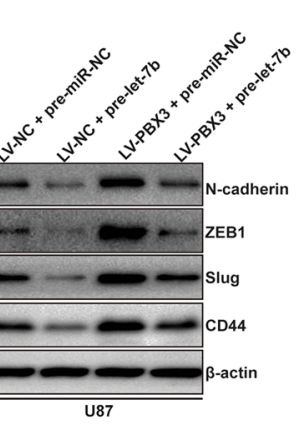

$\mathbf{f}$

$=$ LV-NC + pre-miR-NC

$=\mathrm{LV}-\mathrm{NC}+$ pre-let-7b
$=\mathrm{LV}-\mathrm{PBX} 3+$ pre-miR-NC

- LV-PBX3 + pre-let-7b
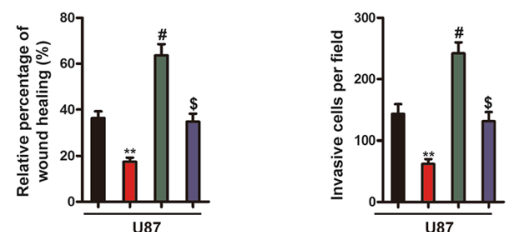

9

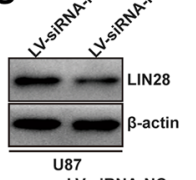

$\begin{aligned} & =\text { LV-siRNA-NC } \\ { }_{8} & =\text { LV-SiRNA-PBX3 }\end{aligned}$
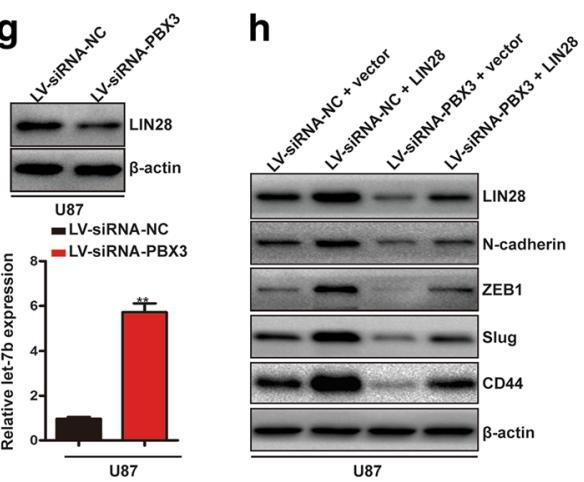

i

i

- LV-siRNA-NC + vector

$=$ LV-SiRNA-NC + LIN28

- LV-siRNA-PBX3 + vector
$=$ LV-SiRNA-PBX $3+$ LIN28
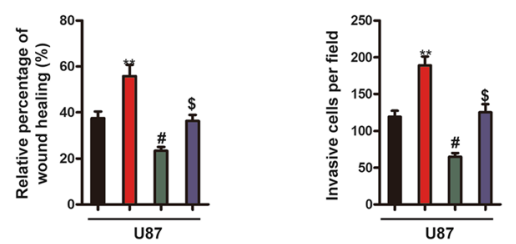

j

- LV-siRNA-NC + anti-miR-NC

- LV-siRNA-NC + anti-let-7b

- LV-siRNA-PBX3 + anti-miR-NC

- LV-cadherin
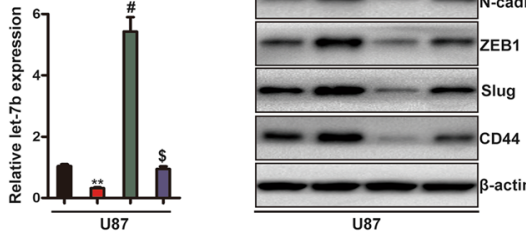

k
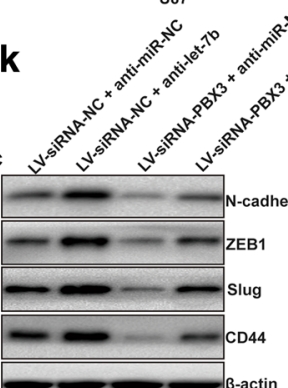

I LV-SiRNA-NC + anti-miR-NC

$=$ LV-siRNA-NC + anti-l-tet-7b

= LV-siRNA-PBX3 + anti-miR-NC
$=$ LV-siRNA-PBX3 + anti-let-7b

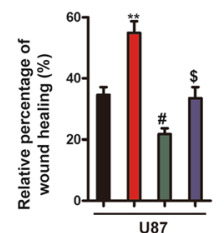

Fig. 3 (See legend on next page.) 


\section{(See figure on previous page.)}

Fig. 3 PBX3 promotes GBM mesenchymal transition, migration and invasion is mediated by LIN28/let-7b axis. a PBX3 overexpression remarkably upregulated LIN28 protein levels and downregulated let-7b expression in U87 cells. b U87 cells stably expressing LV-PBX3 or LV-NC were transfected with siRNA-NC or siRNA-LIN28 and then immunoblotting analysis of LIN28, N-cadherin, ZEB1, Slug and CD44 were performed. $\mathbf{c}$ Quantification of wound-healing (left) and transwell (right) assays. ${ }^{*}$ indicates a statistical significant difference $(p<0.01)$ between LV-NC + siRNANC group and LV-NC + siRNA-LIN28 group. \# indicates a statistical significant difference $(p<0.01)$ between LV-NC + siRNA-NC group and LV-PBX3 + siRNA-NC group. \$ indicates a statistical significant difference $(p<0.01)$ between LV-PBX3 + siRNA-NC group and LV-PBX3 + siRNA-LIN28 group. d U87 cells stably expressing LV-PBX3 or LV-NC were transfected with pre-miR-NC or pre-let-7b and then let-7b expression were determined by qRT-PCR. ${ }^{*}$ indicates a statistical significant difference $(p<0.01)$ between LV-NC + pre-miR-NC group and LV-NC + pre-let-7b group. \# indicates a statistical significant difference $(p<0.01)$ between LV-NC + pre-miR-NC group and LV-PBX3 + pre-miR-NC group. \$ indicates a statistical significant difference $(p<0.01)$ between LV-PBX3 + pre-miR-NC group and LV-PBX3 + pre-let-7b group. e U87 cells stably expressing LV-PBX3 or LV-NC were transfected with pre-miR-NC or pre-let-7b and then immunoblotting analysis of N-cadherin, ZEB1, Slug and CD44 were performed. $\mathbf{f}$

Quantification of wound-healing (left) and transwell (right) assays. ${ }^{* *}$ indicates a statistical significant difference $(p<0.01)$ between LV-NC + premiR-NC group and LV-NC + pre-let-7b group. \# indicates a statistical significant difference $(p<0.01)$ between LV-NC + pre-miR-NC group and LVPBX3 + pre-miR-NC group. \$ indicates a statistical significant difference $(p<0.01)$ between LV-PBX3 + pre-miR-NC group and LV-PBX3 + pre-let-7b group. g PBX3 knockdown remarkably downregulated LIN28 protein levels and upregulated let-7b expression in U87 cells. $\mathbf{h}$ U87 cells stably expressing LV-siRNA-PBX3 or LV-siRNA-NC were transfected with LIN28 overexpressing plasmids or empty vectors and then immunoblotting analysis of LIN28, N-cadherin, ZEB1, Slug and CD44 were performed. i Quantification of wound-healing (left) and transwell (right) assays. **indicates a statistical significant difference $(p<0.01)$ between LV-siRNA-NC + vector group and LV-siRNA-NC + LIN28 group. \# indicates a statistical significant difference $(p<0.01)$ between LV-siRNA-NC + vector group and LV-siRNA-PBX3 + vector group. \$ indicates a statistical significant difference $(p<0.01)$ between LV-siRNA-PBX3 + vector group and LV-siRNA-PBX3 + LIN28 group. $\mathbf{j}$ U87 cells stably expressing LV-siRNAPBX3 or LV-siRNA-NC were transfected with anti-miR-NC or anti-let-7b and then let-7b expression were determined by qRT-PCR. **indicates a statistical significant difference $(p<0.01)$ between LV-siRNA-NC + anti-miR-NC group and LV-siRNA-NC + anti-let-7b group. \# indicates a statistical significant difference $(p<0.01)$ between LV-siRNA-NC + anti-miR-NC group and LV-siRNA-PBX3 + anti-miR-NC group. \$ indicates a statistical significant difference $(p<0.01)$ between LV-siRNA-PBX3 + anti-miR-NC group and LV-siRNA-PBX3 + anti-let-7b group. $\mathbf{k}$ U87 cells stably expressing LV-siRNA-PBX3 or LV-siRNA-NC were transfected with anti-miR-NC or anti-let-7b and then immunoblotting analysis of N-cadherin, ZEB1, Slug and CD44 were performed. I Quantification of wound-healing (left) and transwell (right) assays. ${ }^{* *}$ indicates a statistical significant difference $(p<0.01)$ between LV-siRNA-NC + anti-miR-NC group and LV-siRNA-NC + anti-let-7b group. \# indicates a statistical significant difference $(p<0.01)$ between LV-siRNA-NC + anti-miR-NC group and LV-siRNA-PBX3 + anti-miR-NC group. \$ indicates a statistical significant difference $(p<0.01)$ between LVsiRNA-PBX3 + anti-miR-NC group and LV-siRNA-PBX3 + anti-let-7b group

ERK1/2 pathway in GBM cells. Next, we inhibited MEK/ERK1/2 activation in PBX3-overexpressed cells by U0126 and activated MEK/ERK1/2 pathway in PBX3-downregulated cells by PMA to determine whether PBX3-mediated GBM cell migration, invasion and mesenchymal transition were dependent on MEK/ ERK1/2 pathway. As shown in Fig. 4a, the upregulation of phospho-MEK and phospho-ERK1/2 expression by PBX3 overexpression were significantly reduced by U0126 incubation. In the meantime, the promotive effects of PBX3 on cell migration, invasion and mesenchymal transition were markedly reversed by U0126 treatment (Fig. 4a-c). Consistently, the decreased expression of phospho-MEK and phospho-ERK $1 / 2$ expression by PBX3 downregulation were restored by PMA treatment. Meanwhile, the inhibitory effects after PBX3 downregulation on cell migration, invasion and mesenchymal transition were reversed by PMA incubation (Fig. 4d-f). These results confirmed the hypothesis that PBX3 promotes GBM migration, invasion and mesenchymal transition via activation of MEK/ERK1/2 pathway.

To demonstrate that PBX3 regulates LIN28/let-7b axis via ERK1/2-dependent mechanism. We inhibited ERK1/ 2 signaling in PBX3-overexpressing cells and activated ERK1/2 signaling in PBX3-knockdown cells. As shown in Fig. $5 \mathrm{a}$ and b, ERK1/2 inhibition reversed PBX3 overexpression induced LIN28 upregulation and let-7b downregulation, while ERK1/2 activation restored PBX3 depletion induced LIN28 downregulation and let-7b upregulation. Thus, these data suggest that PBX3 promotes LIN28/let-7b axis via ERK1/2-dependent mechanism. Previous studies have demonstrated that ERK1/2 regulates LIN28/let-7b axis via c-myc-dependent way and c-myc regulates LIN28 expression by directly binding to its promoter [30, 38]. As c-myc plays central roles in GBM progression, including mesenchymal transition [39], we proposed that ERK1/2 signaling promotes LIN28/let-7b axis in GBM cells via c-myc-dependent pathway. To test whether the hypothesis is true and whether the transcriptional factor c-myc might mediated PBX3/ERK1/2-driven mesenchymal transition, we overexpressed c-myc in ERK1/2-inhibited cells and knocked down c-myc in ERK1/2-activated cells. Results showed that LIN28 downregulation and let-7b upregulation induced by ERK1/2 inhibition were prevented by c-myc overexpression (Fig. 5c). Consistently, LIN28 upregulation and let-7b downregulation induced by ERK1/2 activation were reversed by c-myc knockdown (Fig. 5d). Furthermore, ChIP assays confirmed previous studies that c-myc can directly bind to the promoter of LIN28 and this biding was depended on ERK1/2 signaling (Fig. $5 e-g)$. Finally, we found that silencing c-myc can reduced PBX3 overexpression induced increased migration, 
a
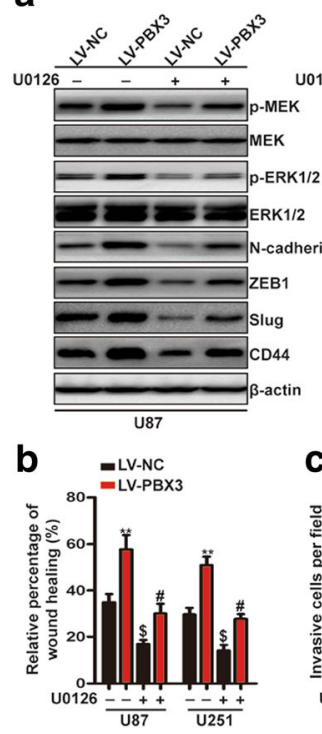
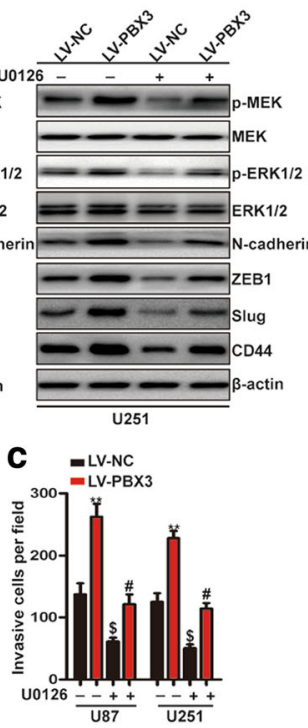
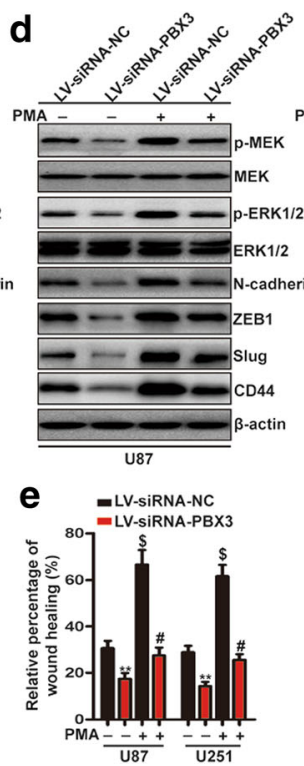
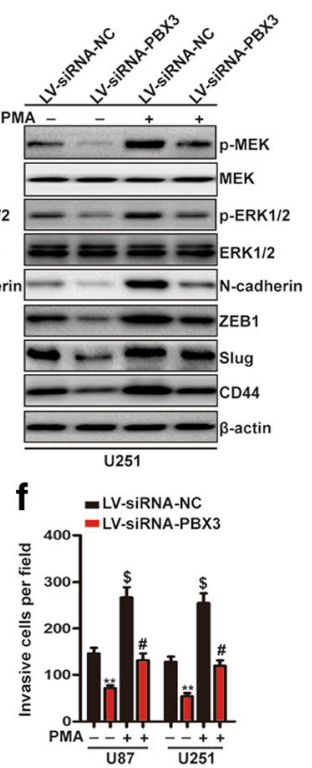

Fig. 4 PBX3 promotes GBM mesenchymal transition, migration and invasion via activating MEK/ERK1/2 signaling pathway. a U87 and U251 cells stably expressing LV-PBX3 or LV-NC were treated with U0126 for $24 \mathrm{~h}$ and immunoblotting analysis of p-MEK, MEK, p-ERK1/2, ERK1/2, N-cadherin, ZEB1, Slug and CD44 were performed. $\mathbf{b}$ and $\mathbf{c}$ Quantification of wound-healing (b) and transwell (c) assays. **indicates a statistical significant difference $(p<0.01)$ between LV-NC + U0126 (-) group and LV-PBX3 + U0126 (-) group. \$ indicates a statistical significant difference $(p<0.01)$ between LV-NC + U0126 (-) group and LV-NC + U0126 (+) group. \# indicates a statistical significant difference $(p<0.01)$ between LV-NC + U0126 (+) group and LV-PBX3 + U0126 (+) group. d U87 and U251 cells stably expressing LV-siRNA-PBX3 or LV-siRNA-NC were treated with PMA for $24 \mathrm{~h}$ and immunoblotting analysis of p-MEK, MEK, p-ERK1/2, ERK1/2, N-cadherin, ZEB1, Slug and CD44 were performed. e and $\mathbf{f}$ Quantification of wound-healing $(\mathbf{e})$ and transwell $(\mathbf{f})$ assays. ${ }^{*}$ indicates a statistical significant difference $(p<0.01)$ between LV-siRNA-NC + PMA $(-)$ group and LVsiRNA-PBX3 + PMA $(-)$ group. \$ indicates a statistical significant difference $(p<0.01)$ between LV-siRNA-NC + PMA $(-)$ group and LV-siRNA-NC + PMA $(+)$ group. \# indicates a statistical significant difference $(p<0.01)$ between LV-siRNA-NC + PMA $(+)$ group and LV-siRNA-PBX3 + PMA

(+) group

invasion and mesenchymal transition, while overexpression of c-myc can restore PBX3 inhibition induced decreased migration, invasion and mesenchymal transition (Additional file 6: Figure S6). Collectively, our finding demonstrated that PBX3 activates LIN28/let-7b axis in GBM cells via ERK1/2-c-myc-dependent mechanism.

\section{Let-7b directly targets PBX3 3'-UTR and forms a positive feedback loop}

Previous studies have demonstrated that PBX3 can be negatively regulated by various miRNAs, including let-7 family members (miR-98, let-7c, and let-7d) [19, 20, 26]. However, whether PBX3 can be regulated by let-7b in GBM remains undetermined. To test whether PBX3 is a direct target of let-7b, luciferase report assays were performed. Fig. 6a shows the predicted interaction between let-7b and the target sites. Relative luciferase activity was significantly inhibited by let-7b when the PBX3 plasmid containing the wild $3^{\prime}$-UTR and mut1 3 '-UTR were present. However, mutations in binding site 2 (mut2) or both the binding sites were mutated abrogated the suppressive effect of let-7b (Fig. 6b). Moreover, western blot analysis showed that let-7d suppressed PBX3 and the downstream targets MEK/ERK1/2, c-myc and LIN28. In contrast, suppression of let-7b enhanced PBX3 and its downstream signaling (Fig. 6c). Taken together, these results suggest that PBX3 is a bona fide target of let-7b and there exists a positive feedback loop between PBX3 and let-7b. Moreover, other targets of let-7 family, which are mesenchymal transition relevant factors, such as HMGA2 and IL-6/STAT3, were also evaluated in our present study. As shown in Fig. 6d, let-7b can also inhibit HMGA2 and IL-6/STAT3 pathways. As PBX3 regulates LIN28/let-7b axis, we wondered whether PBX3 regulates let-7b targets in glioma cells. Therefore, we tested the effect of PBX3 silencing on the expression of let-7b targets. As shown in Fig. 6e, PBX3 silencing decreased the mRNA levels of HMGA2 and IL- 6 and protein ratios of p-STAT3/STAT3 in both U87 and U251 cells. Indeed, our GSEA results showed that hallmark of "IL-6_JAK_STAT3 signaling" is significantly enriched in the patients with high-level of PBX3 (Additional file 7: Figure S7).

We further measured let-7b, HMGA2 and IL-6 mRNA levels in 45 GBM samples and Pearson's correlation analyses were performed. Results showed that let-7b inversely correlated with PBX3, HMGA2 and IL-6 expression (Fig. 6f), supporting that PBX3, HMGA2 and 
a
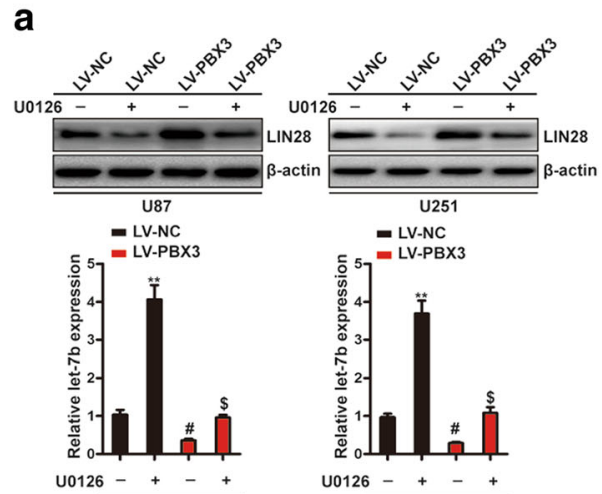

C

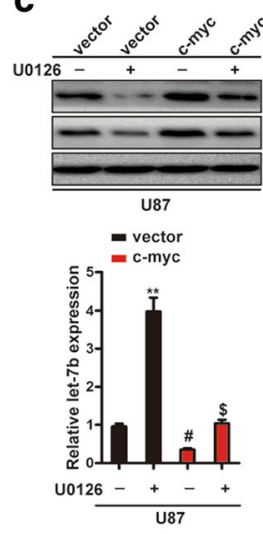

e

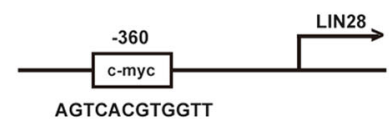

\section{믄 $\lg$}

LIN28 (c-myc)

- WNT5A (c-myc)

CyclinD1 (Jun)
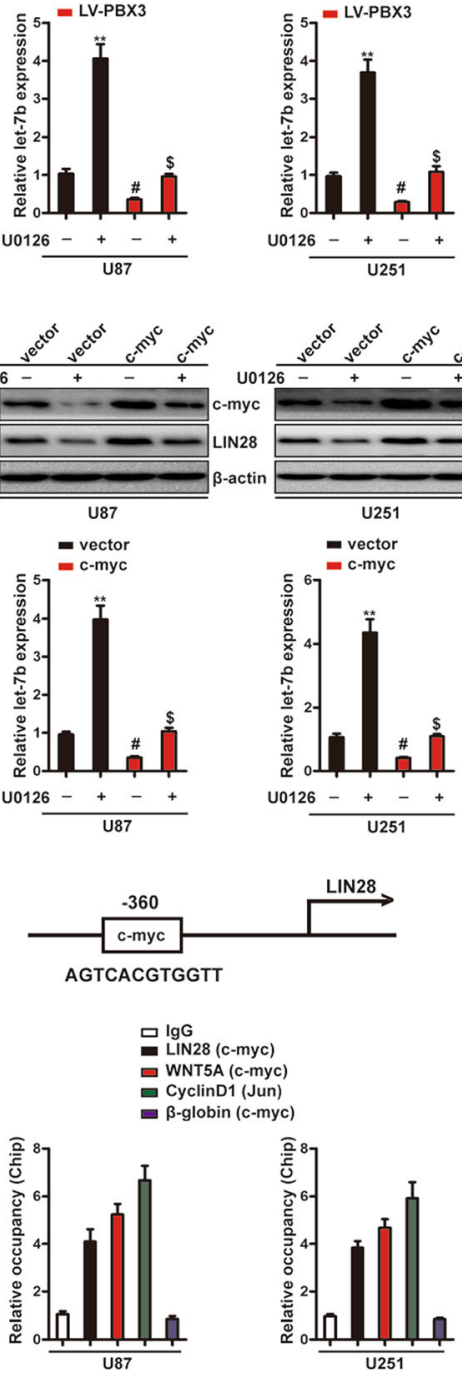

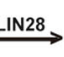

b

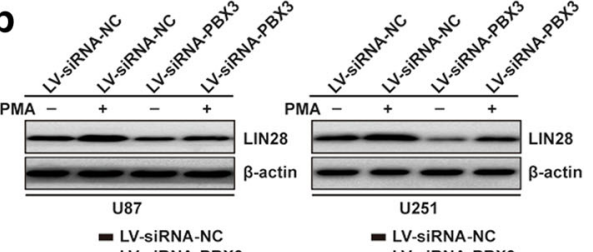

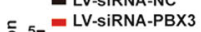

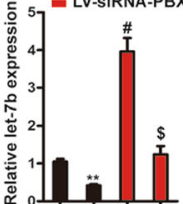

5 ${ }_{5}=$ LV-SiRNA-PBX3

PMA - + +

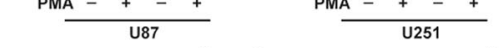

d
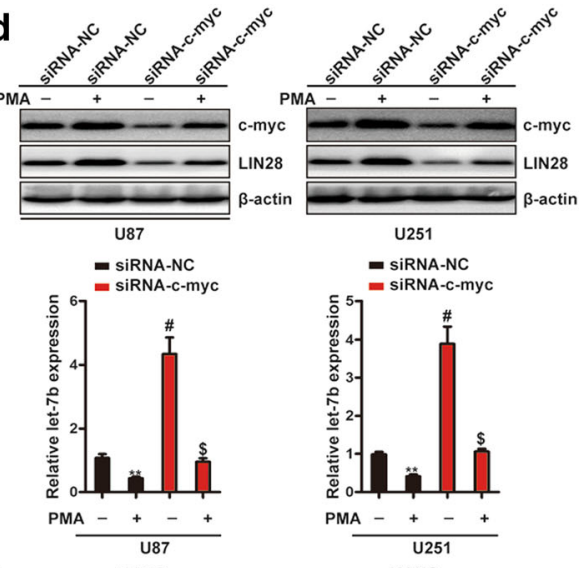

- siRNA-c-myc

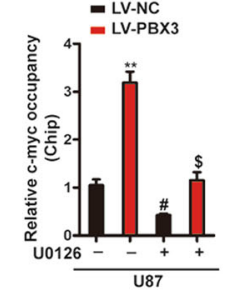

g

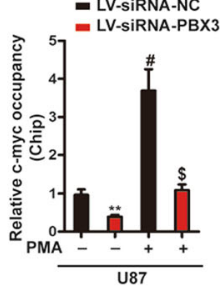

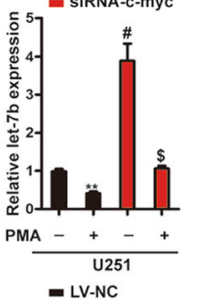

$=$ LV-NC
$=$ LV-PBX3

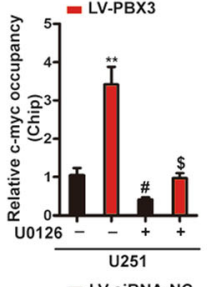

- LV-siRNA-NC

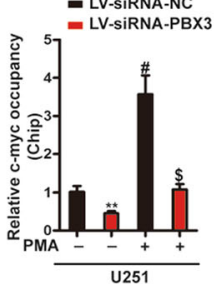

Fig. 5 (See legend on next page.) 
(See figure on previous page.)

Fig. 5 PBX3 activates LIN28/let-7b axis via ERK1/2 and c-myc-dependent mechanisms. a GBM cells stably expressing LV-PBX3 or LV-NC were treated with $\cup 0126$ for $24 \mathrm{~h}$ and then LIN28 protein levels and let-7b expression were determined by western blot and qRT-PCR assays, respectively. ${ }^{*}$ indicates a statistical significant difference $(p<0.01)$ between LV-NC + U0126 (-) group and LV-NC + U0126 (+) group. \# indicates a statistical significant difference $(p<0.01)$ between LV-NC + U0126 (-) group and LV-PBX3 + U0126 $(-)$. \$ indicates a statistical significant difference $(p<0.01)$ between LV-PBX3 + U0126 (-) group and LV-PBX3 + U0126 (+) group. b GBM cells stably expressing LV-siRNA-PBX3 or LV-siRNA-NC were treated with PMA for $24 \mathrm{~h}$ and then LIN28 protein levels and let-7b expression were determined by western blot and qRT-PCR assays, respectively. ${ }^{*}$ indicates a statistical significant difference $(p<0.01)$ between LV-siRNA-NC + PMA $(-)$ group and LV-siRNA-NC + PMA $(+)$ group. \# indicates a statistical significant difference $(p<0.01)$ between LV-siRNA-NC + PMA $(-)$ group and LV-siRNA-PBX3 + PMA $(-)$. \$ indicates a statistical significant difference $(p<0.01)$ between LV-siRNA-PBX3 + PMA $(-)$ group and LV-siRNA-PBX3 + PMA $(+)$ group. c GBM cells infected with c-myc overexpressing plasmids or empty vectors were treated with U0126 for $24 \mathrm{~h}$ and then LIN28 and c-myc protein levels were determined by western blot assays. Let-7b expression were measured by qRT-PCR assays. ${ }^{* *}$ indicates a statistical significant difference $(p<0.01)$ between vector + U0126 (-) group and vector + U0126 (+) group. \# indicates a statistical significant difference $(p<0.01)$ between vector + U0126 (-) group and $c-m y c+$ U0126 (-). \$ indicates a statistical significant difference $(p<0.01)$ between c-myc + U0126 (-) group and c-myc + U0126 (+) group. $\mathbf{d}$ GBM cells infected with siRNA-NC or siRNA-c-myc were treated with PMA for $24 \mathrm{~h}$ and then LIN28 and c-myc protein levels were determined by western blot assays. Let-7b expression were measured by qRT-PCR assays. **indicates a statistical significant difference $(p<0.01)$ between siRNANC + PMA (-) group and siRNA-NC + PMA (+) group. \# indicates a statistical significant difference $(p<0.01)$ between siRNA-NC + PMA $(-)$ group and siRNA-c-myc + PMA $(-)$. \$ indicates a statistical significant difference $(p<0.01)$ between siRNA-c-myc + PMA $(-)$ group and siRNA-c-myc + PMA (+) group. e c-myc regulates LIN28 expression by binding to its promoter in GBM cells. Diagram of amplicons for ChIP-qPCR were illustrated. ChIPs were performed with anti-c-myc antibody and anti-Jun antibody (a positive control). ChIP was analyzed by qRT-PCR, with primers in the LIN28, WNT5A (a positive control), CyclinD1 (a positive control), and $\beta$-globin (a negative control) promoters. $\mathbf{f} \cup 0126$ treatment reversed the effect of PBX3 overexpression-induced enhanced c-myc binding to LIN28 promoters. ${ }^{* *}$ indicates a statistical significant difference $(p<0.01)$ between LV-NC + U0126 (-) group and LV-PBX3 + U0126 (+) group. \# indicates a statistical significant difference $(p<0.01)$ between LV-NC + U0126 (-) group and LV-NC + U0126 (+). \$ indicates a statistical significant difference $(p<0.01)$ between LV-PBX3 + U0126 (-) group and LV-PBX3 + U0126 (+) group. g PMA treatment reversed the effect of PBX3 knockdown-induced decreased c-myc binding to LIN28 promoters. **indicates a statistical significant difference $(p<0.01)$ between LV-siRNA-NC + PMA $(-)$ group and LV-siRNA-PBX3 + PMA $(+)$ group. \# indicates a statistical significant difference $(p<0.01)$ between LV-siRNA-NC + PMA $(-)$ group and LV-siRNA-NC + PMA $(+)$. \$ indicates a statistical significant difference $(p<0.01)$ between LV-siRNA-PBX3 + PMA $(-)$ group and LV-siRNA-PBX3 + PMA $(+)$ group

IL-6 are targets of let-7b. Until now, we established a positive feedback loop between PBX3 and let-7b, which involves MEK/ERK1/2, c-myc and LIN28, and ultimately affects HMGA2 and IL-6/STAT3 pathways to promotes GBM mesenchymal transition, migration and invasion (Fig. 6g).

\section{Downregulation of PBX3 inhibits GBM invasion in vivo}

To further confirm the role of PBX3 in GBM invasion and assess the therapeutic potential of PBX3 depletion in vivo, LV-siRNA-NC- and LV-siRNA-PBX3-transfected U87 cells were intracranially injected into nude mice. As shown in Fig. 7a, downregulation of PBX3 consistently led to decreased tumor invasion compared with the control group. In addition, PBX3 depletion reduced tumor growth and resulted in the formation of significantly smaller tumors when compared with the control group (Fig. $7 \mathrm{~b}$ and $\mathrm{c}$ ). These results demonstrated that targeting PBX3 inhibits GBM invasion and growth in vivo. Next, we performed PBX3, N-cadherin, ZEB1, Slug and CD44 staining to assess the effects of PBX3 on GBM mesenchymal transition. Results showed that inhibition of PBX3 suppressed the expression of $\mathrm{N}$-cadherin, ZEB1, Slug and CD44 (Fig. 7d), indicating that downregulation of PBX3 inhibits GBM mesenchymal transition in vivo. Finally, we investigated whether MEK/ERK1/2/c-myc/ LIN28/let-7b pathway was suppressed upon PBX3 knockdown. Our western blot and qRT-PCR results demonstrated that PBX3 knockdown inhibited p-MEK,
p-ERK1/2, c-myc, LIN28, HMGA2, IL-6 and p-STAT3 protein levels and increased let-7b expression (Fig. 7e and $\mathrm{f}$ ). Taken together, these data support our in vitro findings and reinforce the hypothesis that $\mathrm{PBX} 3$ has a critical role in the maintenance of a mesenchymal and invasive phenotype in human GBM.

\section{Discussion}

Despite aggressive treatment, the prognosis of patients with GBM remains dismal, which is partly caused by the highly invasive nature of GBM [4]. Recently, mesenchymal transition of GBM has been recognized as a key driver of GBM invasion and malignant progression, but signals promote this process are still unclear [7]. Here, we presents evidence for a role of PBX3 in the regulation of the mesenchymal transition program that maintains invasive phenotypes of human GBM. Our demonstrations that PBX3 is upregulated in mesenchymal gliomas compared with proneural gliomas and that it positively correlated with several mesenchymal markers highlight the significance of PBX3 in mesenchymal transition. Notably, we showed that PBX3 actives MEK/ERK1/2 axis, which negatively regulates let-7b by inducing LIN28 expression through oncogenic c-myc transcription. In turn, let-7b inhibits PBX3 expression by directly targeting the 3'-UTR of PBX3. Thus, these data implicate PBX3, MEK/ERK1/2, c-myc and LIN28/let-7b in a positive feedback loop. In addition, PBX3 has been demonstrated to be required for TGF- $\beta$-induced mesenchymal transition, 

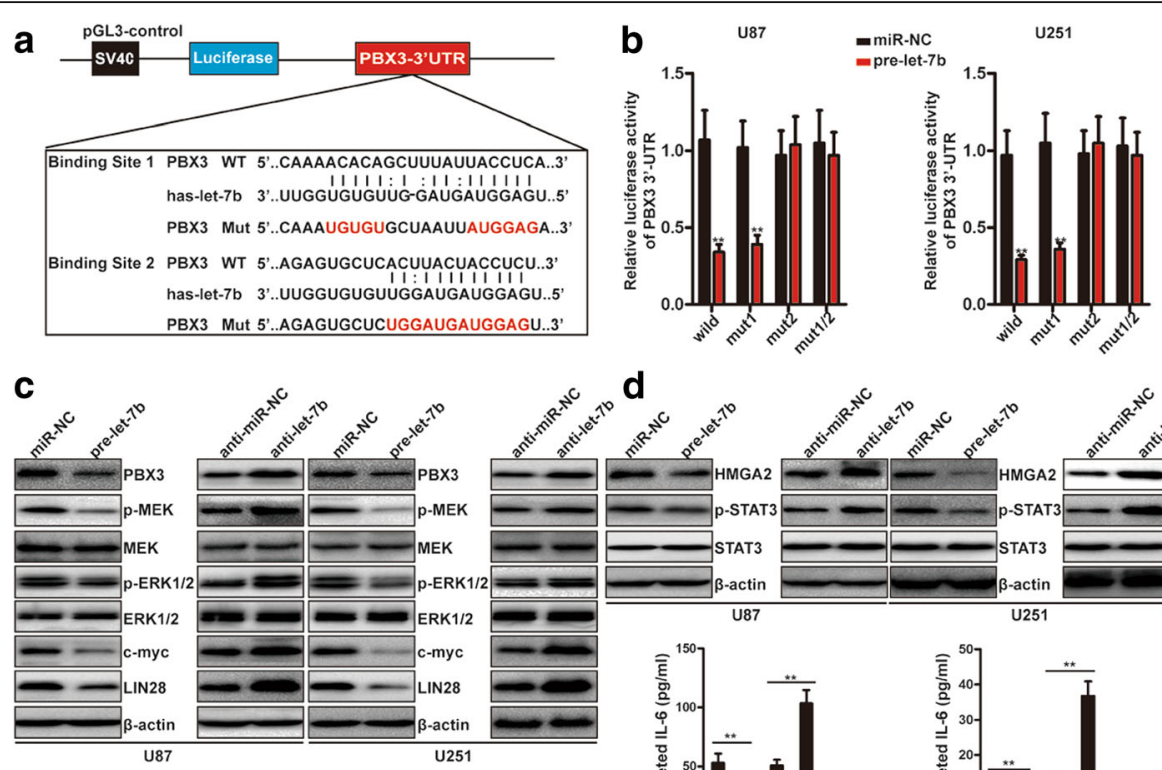

d
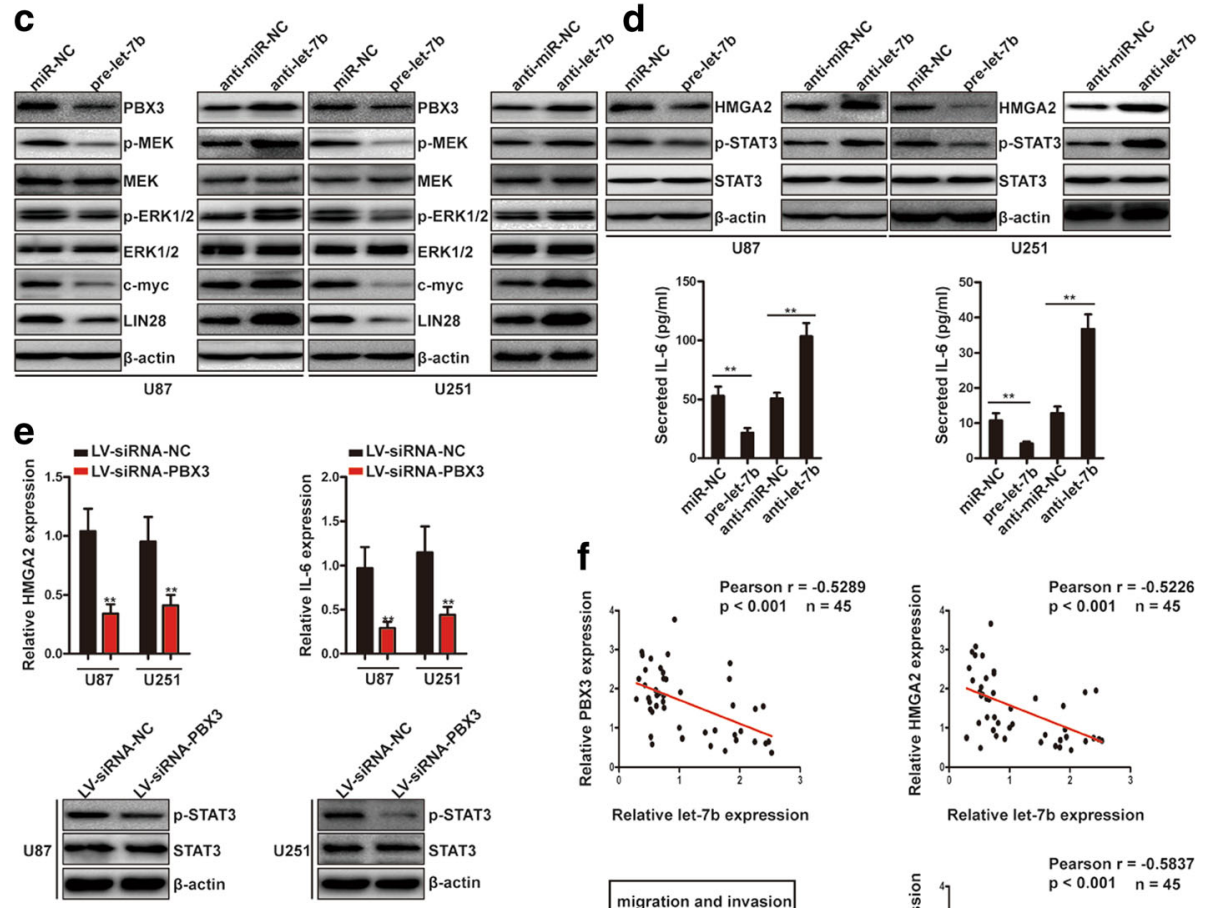

Relative let-7b expression

Relative let-7b expression

g
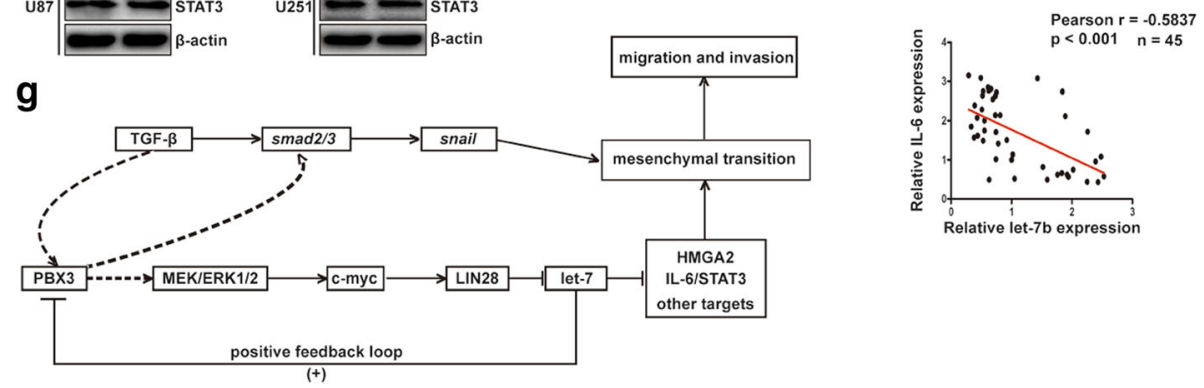

Fig. 6 Let-7b directly targets PBX3 3'-UTR and forms a positive feedback loop. a Predicted binding sites of wild-type (WT) and mutant sequences of let-7b in the $3^{\prime}-$ UTR of PBX3 mRNA. $\mathbf{b}$ Luciferase reporter assays were performed in U87 and U251 cells with co-transfection of indicated WT or mutant 3'-UTR constructs and pre-let-7b mimic or miR-NC mimic. ${ }^{* *} p<0.01$. c Western blot analysis of PBX3, p-MEK, MEK, p-ERK1/2, ERK1/2, cmyc, and LIN28 protein levels in let-7b overexpressing- or depleting-cells. $\mathbf{d}$ Western blot analysis of HMGA2, p-STAT3, and STAT3 protein levels in let-7b overexpressing- or depleting-cells and ELISA analysis of IL-6 protein levels in the supernatants of let-7b overexpressing-or depleting-cells. ${ }^{* *} p<0.01$. e qRT-PCR analysis of HMGA2 and IL-6 mRNA levels and western blot analysis of p-STAT3 and STAT3 protein levels in U87 and U251 cells transfected with LV-siRNA-NC or LV-siRNA-PBX3. f Pearson's correlation analyses indicated that let-7b expression was negatively associated with PBX3, HMGA2 and IL-6 mRNA levels in GBM tissues. g Schematic diagram of the positive feedback loop involves PBX3, MEK/ERK1/2, c-myc and LIN28/let-7b

suggesting that this positive feedback loop may be a part of TGF- $\beta$ signaling. Based on these findings, we proposed that PBX3 could be a promising therapeutic target for preventing GBM mesenchymal transition and invasion.

The members of the PBX family have vital roles in both development and differentiation through regulating gene transcription [40, 41]. Dysregulation of PBX family members has been implicated in various types of human cancers, including GBM $[22,26,27]$. Although our previous studies suggested a role for PBX3 in the biology of GBM proliferation, cell cycle progression, migration and invasion [26, 27], there is a paucity of data defining the 


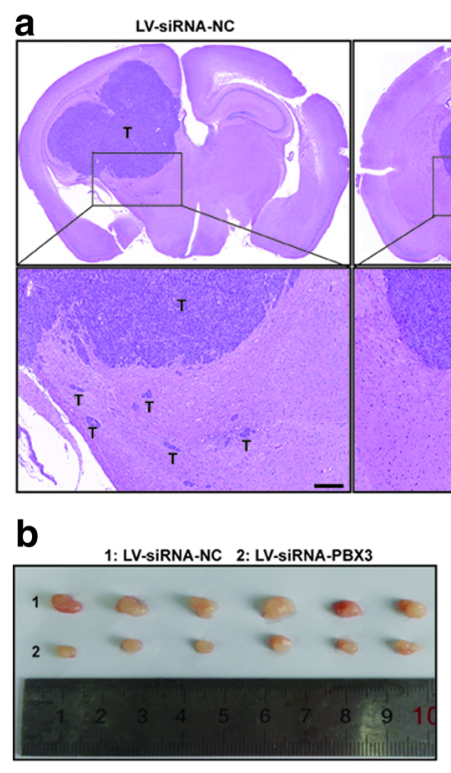

LV-siRNA-PBX3

$\mathbf{e}$

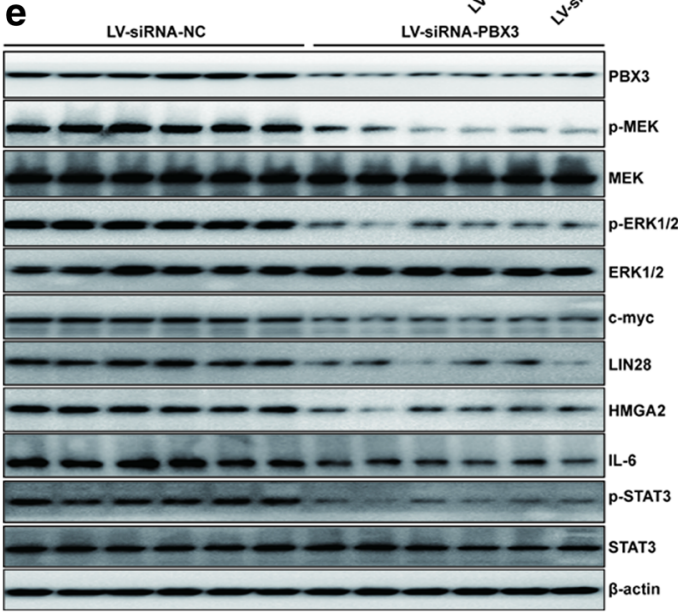

d

H\&E

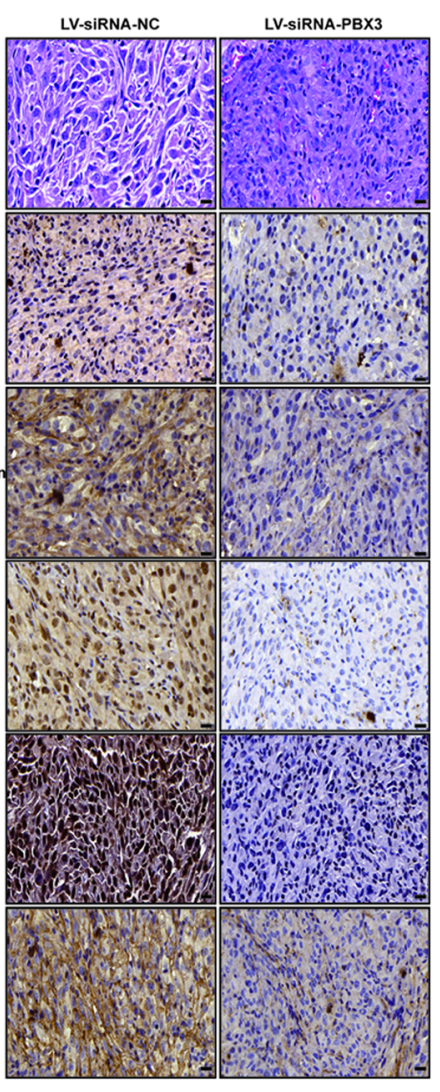

f

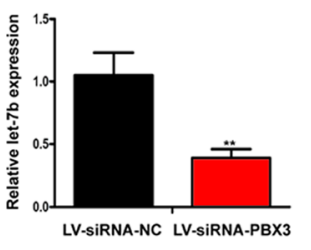

Fig. 7 Targeting PBX3 inhibits GBM invasion in vivo. a Representative images of Hematoxylin and eosin (H\&E) staining of tissues from mice with orthotopic tumors derived from LV-siRNA-NC-U87 or LV-siRNA-PBX3-U87 cells. Scale bar $=500 \mu \mathrm{m}$. $\mathbf{b}$ Images of orthotopic tumors derived from LV-siRNA-NC-U87 or LV-siRNA-PBX3-U87 cells. c The tumors derived from LV-siRNA-NC-U87 or LV-siRNA-PBX3-U87 cells were weighed after imaging. ${ }^{* *} p<0.01$. d H\&E staining and immunohistochemistry for PBX3, N-cadherin, ZEB1, Slug and CD44 in tumors derived from LV-siRNA-NCU87 or LV-siRNA-PBX3-U87 cells. Scale bar $=50 \mu \mathrm{m}$. e Western blot analysis of PBX3, p-MEK, MEK, p-ERK1/2, ERK1/2, c-myc, LIN28, IL-6, p-STAT3, and STAT3 in tumors derived from LV-siRNA-NC-U87 or LV-siRNA-PBX3-U87 cells. $\mathbf{f}$ qRT-PCR analysis of let-7b expression in tumors derived from LV-siRNA-NC-U87 or LV-siRNA-PBX3-U87 cells. ${ }^{* *} p<0.01$

functional role that PBX3 plays in GBM mesenchymal transition. Herein, we first showed that PBX3 is upregulated in mesenchymal gliomas and positively correlated with mesenchymal markers, such as N-cadherin, ZEB1, Slug and CD44. These results suggest that an aberrant increase in PBX3 expression is linked to GBM mesenchymal transition. Furthermore, functional studies showed that PBX3 expression directly impacts GBM invasive phenotypes and mesenchymal transition, as measured by in vitro and in vivo experiments. Our findings is consistent with previous reports that $\mathrm{PBX} 3$ functions as an oncogene and EMT enhancer in other cancers [21, 42, 43].
Although the mesenchymal transition promoting role of PBX3 in GBM has been established, detailed molecular mechanism mediated the role of $\mathrm{PBX} 3$ remains largely unknown. It was recently proposed that PBX3 is a regulator of MEK/ERK1/2 pathway [25]. As activation of MEK/ERK1/2 pathway usually facilitates GBM mesenchymal transition and progression [44], it is reasonable to hypothesis that PBX3 promotes GBM mesenchymal transition via activation of MER/ERK1/2. Indeed, we demonstrated that PBX3 promotes GBM mesenchymal transition was mediated by MEK/ERK1/2 pathway. However, how PBX3 activates MEK/ERK1/2 in GBM remains 
largely unknown. Several activators of MEK/ERK1/2 in human cancers have been identified, such as RAF1 [45], IGF-1R [46] and Connexin-43 [47]. Whether PBX3 activates MEK/ERK1/2 to promote mesenchymal transition through these driving factors remains to be investigated in future.

LIN28 has been shown to be a key RNA-binding protein and plays a critical role in cellular reprogramming and tumor transformation [48]. Overexpression of LIN28 was prevalent in human cancers, including GBM [28]. Moreover, through inhibiting let-7 biogenesis, LIN28 influences let-7 targets translation and promotes malignant progression of cancers [49]. Recent reports showed that the LIN28/let-7 axis plays a critical role in promoting the cancer development, Warburg effect and cancer stem-cell like cells [50-52]. However, the detailed molecular mechanisms involved in regulating LIN28/ let-7 pathway in GBM is still unclear. Several mechanisms that contribute to the dysregulated LIN28 in cancers have been identified in previous studies. For example, miRNAs, such as miR-125b, was reported to inhibit LIN28 in some embryonic stem cells as well as in glioma cells $[17,53]$. Of note, let- 7 miRNAs themselves have also been reported to regulate LIN28 expression, in a feedback manner [50]. Moreover, several transcriptional factors, such as NF- $\mathrm{kB}$ [54], c-myc [30] and $\beta$-catenin [55], can activate LIN28 and repress let-7 expression to augment cancer progression, while REST and ESE3/EHF are transcriptional repressors of LIN28 $[49,51]$. In our present study, we showed that PBX3 can indirectly regulate LIN28 by activating MEK/ERK1/2 axis and then inducing LIN28 expression by transcription factor c-myc, which is consistent with previous reports [30]. The crosstalk between PBX3 and other LIN28 regulators, such as NF- $k B, \beta$-catenin, REST and ESE3/EHF should be investigated in future.

Recent studies reported that c-myc may play a key regulatory role in promoting EMT in many types of cancers [56]. Importantly, c-myc has been reported to regulate miRNAs, which mediate its functions in cancer progression, including EMT. For example, Shao et al. reported that c-myc posttranscriptionally upregulates the expression of PHD finger protein 8 by repressing miR-22 to promote breast cancer EMT [57]. In this study, we demonstrated that c-myc mediates PBX3 induced GBM mesenchymal transition at least partially by repressing let-7b expression. Previous studies have also documented that c-myc inhibits let-7 expression by upregulating let-7 biogenesis inhibitor LIN28 [30], which is consistent with our result. Other miRNAs regulated by c-myc, such as miR-9, miR-23a, miR-15a/16-1, miR-106b, miR-34a, miR-148a and miR-25, are also involved in EMT regulation [58]. Thus, targeting c-myc-induced miRNAs or restoring the expression of c-myc repressed miRNAs seems to be an obvious strategy to combat c-myc-driven EMT. Additional studies will be necessary to identify miRNAs connected to c-myc in GBM progression, especially in GBM mesenchymal transition, for targeted therapy.

TGF- $\beta$ is a master inducer of EMT in cancer cells [59]. In this study, our data indicate that PBX3 represent an important modulator of cell responsiveness to TGF- $\beta$ : PBX3 is necessary for the induction of the mesenchymal transition in response to TGF- $\beta$ in GBM cells. However, the molecular mechanisms by which PBX3 modulates the cellular sensitivity to TGF- $\beta$ remains undetermined in this study. Previous studies have demonstrated that small mother against decapentaplegic (SMAD) signaling elicited by TGF- $\beta$ plays a critical role in TGF- $\beta$ induced EMT [60]. In addition, activated TGF- $\beta$ signaling pathway and high levels of phosphorylation-SMADs were found in human gliomas [61]. Whether PBX3 mediates TGF- $\beta$-induced GBM mesenchymal transition through regulating SMAD signaling will be investigated in our next paper.

\section{Conclusions}

In summary, we identified a PBX3/MEK/ERK1/2/c-myc/ LIN28/let-7b positive feedback loop in GBM cells that induces GBM mesenchymal transition and promotes invasive phenotypes. Thus, PBX3 may serve as a target for prevention and treatment of GBM progression.

\section{Additional files}

\begin{abstract}
Additional file 1: Figure S1. PBX3 is positively correlated with mesenchymal markers. (A-D) Pearson's correlation analyses indicate that PBX3 expression is positively associated with $\mathrm{N}$-cadherin (A), ZEB1 (B), Slug (C), and CD44 (D) expressions in Rembrandt database. (E-H) The expression of N-cadherin (E), ZEB1 (F), Slug (G), and CD44 (H) are increased in GBM samples with high PBX3 expression compared with those with low PBX3 expression. (TIF $1080 \mathrm{~kb}$ )
\end{abstract}

Additional file 2: Figure S2. The effects of PBX3 overexpression or knockdown on glioma cell migration and invasion. (A) Representative images of wound-healing assays using U87 and U251 cells stably expressing LV-NC or LV-PBX3. (B) Quantification of wound-healing assays. ${ }^{* *} p<0.01$. (C) Representative images of transwell assays using U87 and U251 cells stably expressing LV-NC or LV-PBX3. (D) Quantification of transwell assays. ${ }^{* *} p<$ 0.01 . (E) Representative images of wound-healing assays using U87 and U251 cells stably expressing LV-siRNA-NC or LV-siRNA-PBX3. (F) Quantification of wound-healing assays. ${ }^{* *} p<0.01$. (G) Representative images of transwell assays using U87 and U251 cells stably expressing LVsiRNA-NC or LV-siRNA-PBX3. (H) Quantification of transwell assays. ${ }^{* *} p<$ 0.01. (TIF $2002 \mathrm{~kb}$ )

Additional file 3: Figure S3. GBMs with high PBX3 expression were enriched with hallmark of TGF- $\beta$. (TIF $1138 \mathrm{~kb}$ )

Additional file 4: Figure S4. Relative expression of let-7 family members as determined by qRT-PCR in U87 and U251 cells overexpressing LV-NC or LV-PBX3. (TIF 697 kb)

Additional file 5: Figure S5. PBX3 promotes mesenchymal transition, migration and invasion in U251 cells is mediated by LIN28/let-7b axis. (A) PBX3 overexpression remarkably upregulated LIN28 protein levels and downregulated let-7b expression in U251 cells. (B) U251 cells stably 
expressing LV-PBX3 or LV-NC were transfected with siRNA-NC or siRNALIN28 and then immunoblotting analysis of LIN28, N-cadherin, ZEB1, Slug and CD44 were performed. (C) Quantification of wound-healing (left) and transwell (right) assays. **indicates a statistical significant difference $(p<$ 0.01) between LV-NC + siRNA-NC group and LV-NC + siRNA-LIN28 group. \# indicates a statistical significant difference $(p<0.01)$ between LV-NC + siRNA-NC group and LV-PBX3 + siRNA-NC group. \$ indicates a statistical significant difference $(p<0.01)$ between LV-PBX3 + siRNA-NC group and LV-PBX3 + siRNA-LIN28 group. (D) U251 cells stably expressing LV-PBX3 or LV-NC were transfected with pre-miR-NC or pre-let-7b and then let-7b expression were determined by qRT-PCR. ${ }^{*}$ indicates a statistical significant difference $(p<0.01)$ between LV-NC + pre-miR-NC group and LV-NC + prelet-7b group. \# indicates a statistical significant difference $(p<0.01)$ between LV-NC + pre-miR-NC group and LV-PBX3 + pre-miR-NC group. \$ indicates a statistical significant difference $(p<0.01)$ between LV-PBX3 + pre-miR-NC group and LV-PBX3 + pre-let-7b group. (E) U251 cells stably expressing LVPBX3 or LV-NC were transfected with pre-miR-NC or pre-let-7b and then immunoblotting analysis of $\mathrm{N}$-cadherin, ZEB1, Slug and CD44 were performed. (F) Quantification of wound-healing (left) and transwell (right) assays. **indicates a statistical significant difference $(p<0.01)$ between LV-NC + pre-miR-NC group and LV-NC + pre-let-7b group. \# indicates a statistical significant difference $(p<0.01)$ between LV-NC + pre-miR-NC group and LV-PBX3 + pre-miR-NC group. \$ indicates a statistical significant difference $(p<0.01)$ between LV-PBX3 + premiR-NC group and LV-PBX3 + pre-let-7b group. (G) PBX3 knockdown remarkably downregulated LIN28 protein levels and upregulated let$7 \mathrm{~b}$ expression in U251 cells. (H) U251 cells stably expressing LV-siRNA-PBX3 or LV-siRNA-NC were transfected with LIN28 overexpressing plasmids or empty vectors and then immunoblotting analysis of LIN28, N-cadherin, ZEB1, Slug and CD44 were performed. (I) Quantification of wound-healing (left) and transwell (right) assays. **indicates a statistical significant difference $(p<0.01)$ between LV-siRNA-NC + vector group and LV-siRNA-NC + LIN28 group. \# indicates a statistical significant difference $(p<0.01)$ between LVsiRNA-NC + vector group and LV-siRNA-PBX3 + vector group. \$ indicates a statistical significant difference $(p<0.01)$ between LV-siRNA-PBX3 + vector group and LV-siRNA-PBX3 + LIN28 group. (J) U251 cells stably expressing LV-siRNA-PBX3 or LV-siRNA-NC were transfected with anti-miR-NC or antilet-7b and then let-7b expression were determined by qRT-PCR. **indicates a statistical significant difference $(p<0.01)$ between LV-siRNA-NC + antimiR-NC group and LV-siRNA-NC + anti-let-7b group. \# indicates a statistical significant difference $(p<0.01)$ between LV-siRNA-NC + anti-miR-NC group and LV-siRNA-PBX3 + anti-miR-NC group. \$ indicates a statistical significant difference $(p<0.01)$ between LV-siRNA-PBX3 + anti-miR-NC group and LVsiRNA-PBX3 + anti-let-7b group. (K) U251 cells stably expressing LV-siRNAPBX3 or LV-siRNA-NC were transfected with anti-miR-NC or anti-let-7b and then immunoblotting analysis of N-cadherin, ZEB1, Slug and CD44 were performed. (L) Quantification of wound-healing (left) and transwell (right) assays. **indicates a statistical significant difference $(p<0.01)$ between LVsiRNA-NC + anti-miR-NC group and LV-siRNA-NC + anti-let-7b group. \# indicates a statistical significant difference $(p<0.01)$ between LV-siRNA-NC + anti-miR-NC group and LV-siRNA-PBX3 + anti-miR-NC group. $\$$ indicates a statistical significant difference $(p<0.01)$ between LV-siRNA-PBX3 + antimiR-NC group and LV-siRNA-PBX3 + anti-let-7b group. (TIF $2795 \mathrm{~kb}$ )

Additional file 6: Figure S6. Inhibition of c-myc or overexpression of cmyc reversed the PBX3 overexpression- or inhibition-induced GBM cells migration, invasion and mesenchymal transition. (A and B) Quantification of wound-healing and transwell assays. **indicates a statistical significant difference $(p<0.01)$ between LV-NC + siRNA-NC group and LV-NC + siRNA-c-myc group. \# indicates a statistical significant difference $(p<$ 0.01 ) between LV-NC + siRNA-NC group and LV-PBX3 + siRNA-NC group. $\$$ indicates a statistical significant difference $(p<0.01)$ between LV-PBX3 + siRNA-NC group and LV-PBX3 + siRNA-c-myc group. (C) U87 and U251 cells stably expressing LV-PBX3 or LV-NC were transfected with siRNA-NC or siRNA-c-myc and then immunoblotting analysis of c-myc, $\mathrm{N}$-cadherin, ZEB1, Slug and CD44 were performed. (D and E) Quantification of wound-healing and transwell assays. **indicates a statistical significant difference $(p<0.01)$ between LV-siRNA-NC + vector group and LV-siRNA$N C+c$-myc group. \# indicates a statistical significant difference $(p<0.01)$ between LV-siRNA-NC + vector group and LV-siRNA-PBX3 + vector group. \$ indicates a statistical significant difference $(p<0.01)$ between LV-siRNA-PBX3
+ vector group and LV-siRNA-PBX3 + c-myc group. (F) U87 and U251 cells stably expressing LV-siRNA-PBX3 or LV-siRNA-NC were transfected with c-myc overexpressing plasmids or empty vectors and then immunoblotting analysis of c-myc, N-cadherin, ZEB1, Slug and CD44 were performed. (TIF $1118 \mathrm{~kb}$ )

Additional file 7: Figure S7. GBMs with high PBX3 expression were enriched with hallmark of IL-6-JAK-STAT3 signaling. (TIF 1464 kb)

\section{Abbreviations}

TGFB: Transforming growth factor- $\beta$; NF-kB: Nuclear factor $\mathrm{kB}$; ATCC: American Type Culture Collection; BALB: Female Bagg albino; ChIP: Chromatin-immunoprecipitation; DMEM: Dulbecco's modified Eagle's medium; GBM: Glioblastoma; HMGA2: High mobility group A2; NCBIGEO: National Center for Biotechnology Information Gene Expression Omnibus; PBX3: Pre-B-cell leukemia homebox 3; PMA: Phorbol 12-myristate 13-acetate; PMT: Proneural-to-mesenchymal; qRT-PCR: Quantitative real-time polymerase chain reaction; Rembrandt: Repository for Molecular Brain Neoplasia Data; SMAD: Small mother against decapentaplegic; SNAI1: Snail family transcriptional repressor 1; STAT3: Signal transducer and activator of transcription 3; WT: Wild-type

\section{Funding}

This work was funded by the National Natural Science Foundation of China (81300998 and 81471269), Natural Science Foundation of Jiangsu Province (BK20131022 and BK20160047), Jiangsu Province's Key Discipline of Medicine (XK201117), Jiangsu Province and the Priority Academic Program Development of Jiangsu Higher Education Institutions (PAPD), Youth Science Foundation of Suzhou China (KJXW2017038) and the Specially Appointed Professor Foundation of Jiangsu Province (ky216r201307).

\section{Availability of data and materials}

All data generated or analyzed during this study are included either in this article or in the additional files.

\section{Authors' contributions}

XPX and $J$ J designed the experiments, provided direction, and guidance on the whole project. XPX, ZYB and KJ performed and analyzed experiments and prepared the figures. YLL performed GSEA. TLZ, DW and LF drafted the manuscript. NL and JJ reviewed the manuscript and made significant revisions on drafts.

\section{Ethics approval and consent to participate}

All participants provided written informed consent, and the study was approved by the institutional review board and ethics committee of Nanjing Medical University.

\section{Consent for publication}

We have obtained consents to publish this paper from all the participants.

\section{Competing interests}

The authors declare that they have no competing interests.

\section{Publisher's Note}

Springer Nature remains neutral with regard to jurisdictional claims in published maps and institutional affiliations.

\section{Author details}

${ }^{1}$ Department of Neurosurgery, the First Affiliated Hospital of Nanjing Medical University, Guangzhou Road 300, Nanjing, Jiangsu, China. ${ }^{2}$ Department of Neurosurgery, Suzhou Municipal Hospital, Suzhou, Jiangsu, China.

${ }^{3}$ Department of Neurosurgery, Yixing People's Hospital, Yixing, Jiangsu, China.

Received: 6 December 2017 Accepted: 2 May 2018

Published online: 17 July 2018

\section{References}

1. Dolecek TA, Propp JM, Stroup NE, Kruchko C. CBTRUS statistical report: primary brain and central nervous system tumors diagnosed in the United States in 2005-2009. Neuro Oncol. 2012;14(Suppl 5):v1-49. 
2. Stupp R, Mason WP, van den Bent MJ, Weller M, Fisher B, Taphoorn MJ, Belanger K, Brandes AA, Marosi C, Bogdahn U, et al. Radiotherapy plus concomitant and adjuvant temozolomide for glioblastoma. N Engl J Med. 2005;352:987-96.

3. Van Meir EG, Hadjipanayis CG, Norden AD, Shu HK, Wen PY, Olson JJ. Exciting new advances in neuro-oncology: the avenue to a cure for malignant glioma. CA Cancer J Clin. 2010;60:166-93.

4. Lefranc F, Brotchi J, Kiss R. Possible future issues in the treatment of glioblastomas: special emphasis on cell migration and the resistance of migrating glioblastoma cells to apoptosis. J Clin Oncol. 2005;23:2411-22.

5. Onishi M, Ichikawa T, Kurozumi K, Date I. Angiogenesis and invasion in glioma. Brain Tumor Pathol. 2011;28:13-24.

6. Kahlert UD, Nikkhah G, Maciaczyk J. Epithelial-to-mesenchymal(-like) transition as a relevant molecular event in malignant gliomas. Cancer Lett. 2013:331:131-8.

7. Wang $Y$, Jiang T. Understanding high grade glioma: molecular mechanism, therapy and comprehensive management. Cancer Lett. 2013;331:139-46.

8. Li A, Walling J, Ahn S, Kotliarov Y, Su Q, Quezado M, Oberholtzer JC, Park J, Zenklusen JC, Fine HA. Unsupervised analysis of transcriptomic profiles reveals six glioma subtypes. Cancer Res. 2009;69:2091-9.

9. Huse JT, Phillips HS, Brennan CW. Molecular subclassification of diffuse gliomas: seeing order in the chaos. Glia. 2011;59:1190-9.

10. Phillips HS, Kharbanda S, Chen R, Forrest WF, Soriano RH, Wu TD, Misra A, Nigro JM, Colman H, Soroceanu L, et al. Molecular subclasses of high-grade glioma predict prognosis, delineate a pattern of disease progression, and resemble stages in neurogenesis. Cancer Cell. 2006;9:157-73.

11. Verhaak RG, Hoadley KA, Purdom E, Wang V, Qi Y, Wilkerson MD, Miller CR Ding L, Golub T, Mesirov JP, et al. Integrated genomic analysis identifies clinically relevant subtypes of glioblastoma characterized by abnormalities in PDGFRA, IDH1, EGFR, and NF1. Cancer Cell. 2010;17:98-110.

12. Bhat KPL, Balasubramaniyan V, Vaillant B, Ezhilarasan R, Hummelink K, Hollingsworth F, Wani K, Heathcock L, James JD, Goodman LD, et al. Mesenchymal differentiation mediated by NF-kappaB promotes radiation resistance in glioblastoma. Cancer Cell. 2013;24:331-46.

13. Carro MS, Lim WK, Alvarez MJ, Bollo RJ, Zhao X, Snyder EY, Sulman EP, Anne $\mathrm{SL}$, Doetsch F, Colman $\mathrm{H}$, et al. The transcriptional network for mesenchymal transformation of brain tumours. Nature. 2010;463:318-25.

14. van den Boom J, Wolter M, Kuick R, Misek DE, Youkilis AS, Wechsler DS, Sommer C, Reifenberger G, Hanash SM. Characterization of gene expression profiles associated with glioma progression using oligonucleotide-based microarray analysis and real-time reverse transcription-polymerase chain reaction. Am J Pathol. 2003:163:1033-43.

15. Lau J, Ilkhanizadeh S, Wang S, Miroshnikova YA, Salvatierra NA, Wong RA, Schmidt C, Weaver VM, Weiss WA, Persson AI. STAT3 blockade inhibits radiationinduced malignant progression in glioma. Cancer Res. 2015;75:4302-11.

16. Kim SH, Ezhilarasan R, Phillips E, Gallego-Perez D, Sparks A, Taylor D, Ladner K, Furuta T, Sabit H, Chhipa R, et al. Serine/threonine kinase MLK4 determines mesenchymal identity in glioma stem cells in an NF-kappaBdependent manner. Cancer Cell. 2016;29:201-13.

17. Mahabir R, Tanino M, Elmansuri A, Wang L, Kimura T, Itoh T, Ohba Y, Nishihara H, Shirato H, Tsuda M, Tanaka S. Sustained elevation of snail promotes glial-mesenchymal transition after irradiation in malignant glioma. Neuro-Oncology. 2014;16:671-85.

18. Monica K, Galili N, Nourse J, Saltman D, Cleary ML. PBX2 and PBX3, new homeobox genes with extensive homology to the human proto-oncogene PBX1. Mol Cell Biol. 1991;11:6149-57.

19. Ramberg H, Alshbib A, Berge V, Svindland A, Tasken KA. Regulation of PBX3 expression by androgen and let-7d in prostate cancer. Mol Cancer. 2011;10:50.

20. Han HB, Gu J, Zuo HJ, Chen ZG, Zhao W, Li M, Ji DB, Lu YY, Zhang ZQ. Let7c functions as a metastasis suppressor by targeting MMP11 and PBX3 in colorectal cancer. J Pathol. 2012;226:544-55.

21. Li B, Zhang S, Shen H, Li C. MicroRNA-144-3p suppresses gastric cancer progression by inhibiting epithelial-to-mesenchymal transition through targeting PBX3. Biochem Biophys Res Commun. 2017:484:241-7.

22. Han H, Du Y, Zhao W, Li S, Chen D, Zhang J, Liu J, Suo Z, Bian X, Xing B, Zhang Z. PBX3 is targeted by multiple miRNAs and is essential for liver tumour-initiating cells. Nat Commun. 2015;6:8271.

23. Yu T, Zhang $X$, Zhang $L$, Wang $Y$, Pan $H$, Xu Z, Pang X. MicroRNA-497 suppresses cell proliferation and induces apoptosis through targeting PBX3 in human multiple myeloma. Am J Cancer Res. 2016;6:2880-9.
24. Guo H, Chu Y, Wang L, Chen X, Chen Y, Cheng H, Zhang L, Zhou Y, Yang FC, Cheng T, et al. PBX3 is essential for leukemia stem cell maintenance in MLL-rearranged leukemia. Int J Cancer. 2017;141:324-35.

25. Han HB, Gu J, Ji DB, Li ZW, Zhang Y, Zhao W, Wang LM, Zhang ZQ. PBX3 promotes migration and invasion of colorectal cancer cells via activation of MAPKJERK signaling pathway. World J Gastroenterol. 2014;20:18260-70.

26. Xu X, Bao Z, Liu Y, Ji J, Liu N. MicroRNA-98 attenuates cell migration and invasion in glioma by directly targeting pre-B cell leukemia Homeobox 3. Cell Mol Neurobiol. 2017;37(8):1359-71.

27. Xu X, Cai N, Bao Z, You Y, Ji J, Liu N. Silencing pre-B-cell leukemia homeobox 3 decreases the proliferation of human glioma cells in vitro and in vivo. J Neuro-Oncol. 2017;135(3):453-63.

28. Qin R, Zhou J, Chen C, Xu T, Yan Y, Ma Y, Zheng Z, Shen Y, Lu Y, Fu D, Chen J. LIN28 is involved in glioma carcinogenesis and predicts outcomes of glioblastoma multiforme patients. PLoS One. 2014;9:e86446.

29. Liu Y, Li H, Feng J, Cui X, Huang W, Li Y, Su F, Liu Q, Zhu J, Lv X, et al. Lin28 induces epithelial-to-mesenchymal transition and stemness via downregulation of let-7a in breast cancer cells. PLoS One. 2013;8:e83083.

30. Dangi-Garimella S, Yun J, Eves EM, Newman M, Erkeland SJ, Hammond SM, Minn AJ, Rosner MR. Raf kinase inhibitory protein suppresses a metastasis signalling cascade involving LIN28 and let-7. EMBO J. 2009;28:347-58.

31. Martin PM, Aeder SE, Chrestensen CA, Sturgill TW, Hussaini IM. Phorbol 12myristate 13-acetate and serum synergize to promote rapamycin-insensitive cell proliferation via protein kinase C-eta. Oncogene. 2007;26:407-14.

32. Praveen Kumar VR, Sehgal $P$, Thota B, Patil S, Santosh $V$, Kondaiah P. Insulin like growth factor binding protein 4 promotes GBM progression and regulates key factors involved in EMT and invasion. J Neuro-Oncol. 2014;116:455-64.

33. Zhang L, Zhang W, Li Y, Alvarez A, Li Z, Wang Y, Song L, Lv D, Nakano I, Hu B, et al. SHP-2-upregulated ZEB1 is important for PDGFRalpha-driven glioma epithelial-mesenchymal transition and invasion in mice and humans. Oncogene. 2016:35:5641-52.

34. Cheng WY, Kandel JJ, Yamashiro DJ, Canoll P, Anastassiou D. A multi-cancer mesenchymal transition gene expression signature is associated with prolonged time to recurrence in glioblastoma. PLoS One. 2012;7:e34705.

35. Srivastava C, Irshad K, Dikshit B, Chattopadhyay P, Sarkar C, Gupta DK, Sinha $\mathrm{S}$, Chosdol K. FAT1 modulates EMT and stemness genes expression in hypoxic glioblastoma. Int J Cancer. 2018;142:805-12.

36. Joseph JV, Conroy S, Tomar T, Eggens-Meijer E, Bhat K, Copray S, Walenkamp AM, Boddeke E, Balasubramanyian V, Wagemakers $M$, et al. TGF-beta is an inducer of ZEB1-dependent mesenchymal transdifferentiation in glioblastoma that is associated with tumor invasion. Cell Death Dis. 2014;5:e1443.

37. Zheng Y, Miu Y, Yang X, Yang X, Zhu M. CCR7 mediates TGF-beta1-induced human malignant glioma invasion, migration, and epithelial-mesenchymal transition by activating MMP2/9 through the nuclear factor KappaB signaling pathway. DNA Cell Biol. 2017;36:853-61.

38. Manier S, Powers JT, Sacco A, Glavey SV, Huynh D, Reagan MR, Salem KZ, Moschetta M, Shi J, Mishima Y, et al. The LIN28B/let-7 axis is a novel therapeutic pathway in multiple myeloma. Leukemia. 2017;31:853-60.

39. Song $Y$, Hu Z, Long H, Peng Y, Zhang X, Que T, Zheng S, Li Z, Wang $G, Y i L$, et al. A complex mechanism for HDGF-mediated cell growth, migration, invasion, and TMZ chemosensitivity in glioma. J Neuro-Oncol. 2014;119:285-95.

40. Rhee JW, Arata A, Selleri L, Jacobs Y, Arata S, Onimaru H, Cleary ML. Pbx3 deficiency results in central hypoventilation. Am J Pathol. 2004; 165:1343-50.

41. Toresson H, Parmar M, Campbell K. Expression of Meis and Pbx genes and their protein products in the developing telencephalon: implications for regional differentiation. Mech Dev. 2000;94:183-7.

42. Lamprecht S, Kaller M, Schmidt EM, Blaj C, Schiergens TS, Engel J, Jung A, Hermeking H, Grunewald TGP, Kirchner T, Horst D. PBX3 is part of an EMT regulatory network and indicates poor outcome in colorectal Cancer. Clin Cancer Res. 2018;24(8):1974-86.

43. Wang S, Li C, Wang W, Xing C. PBX3 promotes gastric cancer invasion and metastasis by inducing epithelial-mesenchymal transition. Oncol Lett. 2016; 12:3485-91.

44. Huang S, Liu F, Niu Q, Li Y, Liu C, Zhang L, Ni D, Pu X. GLIPR-2 overexpression in HK-2 cells promotes cell EMT and migration through ERK1/2 activation. PLoS One. 2013:8:e58574.

45. Chen L, Wang Q, Wang GD, Wang HS, Huang Y, Liu XM, Cai XH. miR-16 inhibits cell proliferation by targeting IGF1R and the Raf1-MEK1/2-ERK1/2 pathway in osteosarcoma. FEBS Lett. 2013;587:1366-72. 
46. Shi ZM, Wang XF, Qian X, Tao T, Wang L, Chen QD, Wang XR, Cao L, Wang YY, Zhang JX, et al. MiRNA-181b suppresses IGF-1R and functions as a tumor suppressor gene in gliomas. RNA. 2013;19:552-60.

47. Yamada A, Futagi M, Fukumoto E, Saito K, Yoshizaki K, Ishikawa M, Arakaki $M$, Hino R, Sugawara $Y$, Ishikawa $M$, et al. Connexin 43 is necessary for salivary gland branching morphogenesis and FGF10-induced ERK1/2 phosphorylation. J Biol Chem. 2016;291:904-12.

48. Jiang S, Baltimore D. RNA-binding protein Lin28 in cancer and immunity. Cancer Lett. 2016;375:108-13.

49. Balzeau J, Menezes MR, Cao S, Hagan JP. The LIN28/let-7 pathway in Cancer. Front Genet. 2017:8:31.

50. Farzaneh M, Attari F, Khoshnam SE. Concise review: LIN28/let-7 signaling, a critical double-negative feedback loop during pluripotency, reprogramming, and Tumorigenicity. Cell Reprogram. 2017;19:289-93.

51. Albino D, Civenni G, Dallavalle C, Roos M, Jahns H, Curti L, Rossi S, Pinton S, D'Ambrosio G, Sessa F, et al. Activation of the Lin28/let-7 Axis by loss of ESE3/EHF promotes a tumorigenic and stem-like phenotype in prostate Cancer. Cancer Res. 2016;76:3629-43.

52. Ma X, Li C, Sun L, Huang D, Li T, He X, Wu G, Yang Z, Zhong X, Song L, et al. Lin28/let-7 axis regulates aerobic glycolysis and cancer progression via PDK1. Nat Commun. 2014;5:5212.

53. Wang J, Cao N, Yuan M, Cui H, Tang Y, Qin L, Huang X, Shen N, Yang HT. MicroRNA-125b/Lin28 pathway contributes to the mesendodermal fate decision of embryonic stem cells. Stem Cells Dev. 2012;21:1524-37.

54. Iliopoulos D, Hirsch HA, Struhl K. An epigenetic switch involving NF-kappaB, Lin28, Let-7 MicroRNA, and IL6 links inflammation to cell transformation. Cell. 2009;139:693-706.

55. Cai WY, Wei TZ, Luo QC, Wu QW, Liu QF, Yang M, Ye GD, Wu JF, Chen YY, Sun GB, et al. The Wnt-beta-catenin pathway represses let-7 microRNA expression through transactivation of Lin28 to augment breast cancer stem cell expansion. J Cell Sci. 2013;126:2877-89.

56. Zhao B, Liu L, Mao J, Zhang Z, Wang Q, Li Q. PIM1 mediates epithelialmesenchymal transition by targeting Smads and c-Myc in the nucleus and potentiates clear-cell renal-cell carcinoma oncogenesis. Cell Death Dis. 2018;9:307.

57. Shao P, Liu Q, Maina PK, Cui J, Bair TB, Li T, Umesalma S, Zhang W, Qi HH. Histone demethylase PHF8 promotes epithelial to mesenchymal transition and breast tumorigenesis. Nucleic Acids Res. 2017:45:1687-702.

58. Jackstadt R, Hermeking H. MicroRNAs as regulators and mediators of c-MYC function. Biochim Biophys Acta. 2015:1849:544-53.

59. Moustakas A, Heldin CH. Mechanisms of TGFbeta-induced epithelialmesenchymal transition. J Clin Med. 2016;5:63.

60. Xu M, He J, Li J, Feng W, Zhou H, Wei H, Zhou M, Lu Y, Zeng J, Peng W, et al. Methyl-CpG-binding domain 3 inhibits epithelial-mesenchymal transition in pancreatic cancer cells via TGF-beta/Smad signalling. Br J Cancer. 2017; 116:91-9.

61. Wang H, Pan JQ, Luo L, Ning XJ, Ye ZP, Yu Z, Li WS. NF-kappaB induces miR-148a to sustain TGF-beta/Smad signaling activation in glioblastoma. Mol Cancer. 2015;14:2

Ready to submit your research? Choose BMC and benefit from:

- fast, convenient online submission

- thorough peer review by experienced researchers in your field

- rapid publication on acceptance

- support for research data, including large and complex data types

- gold Open Access which fosters wider collaboration and increased citations

- maximum visibility for your research: over $100 \mathrm{M}$ website views per year

At BMC, research is always in progress.

Learn more biomedcentral.com/submissions 